\title{
De novo sequencing of tree peony (Paeonia suffruticosa) transcriptome to identify critical genes involved in flowering and floral organ development
}

Shunli Wang ${ }^{1,2+}$, Jie Gao ${ }^{1,2+}$, Jingqi Xue $e^{1,2 \dagger}$, Yuqian Xue ${ }^{1,2}$, Dandan $\mathrm{Li}^{1,2}$, Yanren Guan ${ }^{1,2}$ and Xiuxin Zhang ${ }^{1,2^{*}}$

\begin{abstract}
Background: Tree peony (Paeonia suffruticosa Andrews) is a globally famous ornamental flower, with large and colorful flowers and abundant flower types. However, a relatively short and uniform flowering period hinders the applications and production of ornamental tree peony. Unfortunately, the molecular mechanism of regulating flowering time and floral organ development in tree peony has yet to be elucidated. Because of the absence of genomic information, 454-based transcriptome sequence technology for de novo transcriptomics was used to identify the critical flowering genes using re-blooming, non-re-blooming, and wild species of tree peonies.
\end{abstract}

Results: A total of 29,275 unigenes were obtained from the bud transcriptome, with an N50 of $776 \mathrm{bp}$. The average length of unigenes was $677.18 \mathrm{bp}$, and the longest sequence was $5815 \mathrm{bp}$. Functional annotation showed that 22,823, 17,321, 13,312, 20,041, and 9940 unigenes were annotated by NCBI-NR, Swiss-Prot, COG, GO, and KEGG, respectively. Within the differentially expressed genes (DEGs) 64 flowering-related genes were identified and some important flowering genes were also characterized by bioinformatics methods, reverse transcript polymerase chain reaction (RT-PCR), and rapid-amplification of CDNA ends (RACE). Then, the putative genetic network of flowering induction pathways and a floral organ development model were put forward, according to the comparisons of DEGs in any two samples and expression levels of the important flowering genes in differentiated buds, buds from different developmental stages, and with GA or vernalization treated. In tree peony, five pathways (long day, vernalization, autonomous, age, and gibberellin) regulated flowering, and the floral organ development followed an ABCE model. Moreover, it was also found that the genes PSAP1, PSCOL1, PSCRY1, PSCRY2, PSFT, PSLFY, PSLHY, PSGI, PSSOC1, and PSVIN3 probably regulated re-blooming of tree peony.

Conclusion: This study provides a comprehensive report on the flowering-related genes in tree peony for the first time and investigated the expression levels of the critical flowering related genes in buds of different cultivars, developmental stages, differentiated primordium, and flower parts. These results could provide valuable insights into the molecular mechanisms of flowering time regulation and floral organ development.

Keywords: Tree peony, Transcriptome, Flowering induction pathway, Floral model, Re-blooming, MADS-box gene

\footnotetext{
* Correspondence: zhangxiuxin@caas.cn

†Shunli Wang, Jie Gao and Jingqi Xue contributed equally to this work.

'Key Laboratory of Biology and Genetic Improvement of Horticultural Crops,

Ministry of Agriculture and Rural Affairs, Beijing, People's Republic of China

${ }^{2}$ Institute of Vegetables and Flowers, Chinese Academy of Agricultural

Science, Institute of Peony, Chinese Academy of Agricultural Science, Beijing

100081, China
}

(c) The Author(s). 2019 Open Access This article is distributed under the terms of the Creative Commons Attribution 4.0 International License (http://creativecommons.org/licenses/by/4.0/), which permits unrestricted use, distribution, and reproduction in any medium, provided you give appropriate credit to the original author(s) and the source, provide a link to the Creative Commons license, and indicate if changes were made. The Creative Commons Public Domain Dedication waiver (http://creativecommons.org/publicdomain/zero/1.0/) applies to the data made available in this article, unless otherwise stated. 


\section{Background}

Tree peony (Paeonia suffruticosa Andrews) belongs to section Moutan DC of the genus Paeonia and family Paeoniaceae and is the first candidate for China's national flower. Tree peony is valued all over the world due to its large and colorful flowers $[1,2]$. There are nine wild species of tree peony, $P$. suffruticosa, $P$. cathayana, $P$. jishanensis, $P$. qiui, $P$. ostii, $P$. rockii, $P$. decomposita, $P$. delavayi, and $P$. ludlowii, and more than 2000 cultivars of $P$. suffruticosa worldwide have been produced using conventional breeding [1-3]. The origin of the most important garden ornamental cultivars in China is a result of homoploid hybridization between $P$. ostii, $P$. qiui, $P$. rockii, $P$. jishanensis, and $P$. cathayana species, while the new varieties with colorful flowers from cultivation of $P$. lutea and $P$. suffruticosa were the result of tree peony breeding breakthroughs since 1997 (Martin, 1997; Zhou et al. 2014) . Now, tree peony cultivars can be geographically classified into seven worldwide groups: (1) Chinese Zhongyuan cultivars, (2) Chinese Xibei cultivars, (3) Chinese Xinan cultivars, (4) Chinese Jiangnan cultivars, (5) European cultivars, (6) American cultivars, and (7) Japanese cultivars [1]. Flowering times differ among different cultivars. Generally, the flowering time of Chinese cultivars is earlier than that of Japanese cultivars, and European cultivars and American cultivars are relatively late, having the same flowering time as $P$. delavayi and $P$. ludlowii. The different flowering time and long flowering period are very important for applications and potted production of tree peony. Thus, understanding of the molecular mechanism of flowering time in tree peony could provide a theoretical basis for flowering regulation and breeding.

In Arabidopsis, flowering at the right time is ensured by an intricate regulatory network that has evolved in response to a diverse range of environmental and internal signals. More than 80 genes that regulate flowering time have been identified by genetic and physiological analysis of flowering time in Arabidopsis [4]. Regulation occurs through well-established flowering genetic pathways, such as photoperiod, vernalization, gibberellins (GA), age, autonomous, and thermosensory pathways [5-8]. FLOWERING LOCUS T (FT), SUPPERSSOR OF CONSTANS OF OVEREXPRESSION1 (SOC1), and LEAFY $(L F Y)$ are considered integrating factors in these pathways and are located downstream of FLOWERING LOCUS C (FLC) and CONSTANS (CO) genes, which regulate flowering time by integrating different flowering signals $[8,9]$.

Timely flowering determines the commercial value of tree peonies. In the past decade, forcing culture technology and re-blooming in autumn was first investigated to achieve tree peony flowering at the proper time. These studies focused on cultivar selection, physiological change, chilling effect, and hormone analysis $[1,2,10-12]$. The effects of exogenous $\mathrm{GA}_{3}$ on flowering quality, endogenous hormones, and hormone- and flowering-associated gene expression in a forcing culture of tree peony were also deciphered [13]. Endo-dormancy-imposed growth arrest is one of the key characteristics preventing tree peony from flowering well. Huang et al. [14] and Gai et al. [15] used a subtractive cDNA library and transcriptome sequencing, respectively, to identify key genes associated with the release of dormant buds in tree peony; genes included PsII, PsMPT, GA2, GA20ox, GA2ox, RGA1, SPINDLY (SPY), and AMY2. PsFT, PsVIN3, $P s C O$, and PsGA20ox were identified to play important roles in the regulation of re-blooming in tree peony by transcriptome sequencing [16]. According to the reported transcriptome results, some functional genes related to flowering, including SHORT VEGETATIVE PHASE (SVP), SQUAMOSA PROMOTER BINDING PROTEIN LIKE 9 (SPL9), and SOC1, have also been cloned [1, 2, 12]. However, the detailed mechanism of the flowering induction pathway is unclear in tree peony, which affects the improvement of the quality of the forcing culture of tree peony.

RNA-seq is a recently developed approach for profiling transcriptomes [17] that has many advantages including being cost-effective, highly sensitive, accurate, and having a large dynamic range. Due to these advantages, RNA-seq is now widely used to analyze gene expression, discover novel transcripts, decipher the molecular mechanism of regulated development and growth, and develop SNP and SSR markers [16-23]. In particular, it has been a powerful tool for analysis of species that lack reference genome information [24].

In this study, we described the utilization of 454based transcriptome sequencing technology for de novo transcriptomics to identify the critical floweringrelated genes using reblooming, non-re-blooming, and wild species of tree peonies. We obtained 29,275 unigenes, including 64 flowering-related genes, and proposed a flowering induction pathway and floral organ development model by analysis of differentially expressed genes (DEGs) between any two samples. Then, the critical flowering-related genes were also selected to do expression analysis in different tree peony cultivars, and buds at different developmental stages or under different treatments; the results validated the postulated flowering induction pathway and floral organ development model. At the same time, ten candidate re-blooming genes were also identified. Our results provide valuable insights into the molecular mechanisms of flowering time regulation and floral organ development of tree peony. 


\section{Results}

\section{GS-FLX sequencing and a de novo assembled tree} peony transcriptome

Using 454 sequencing, 31,505 contigs with 20,667,433 total residues were obtained. These contigs were further assembled into 29,275 unigenes, with 19,824 total residues of $498 \mathrm{bp}$ and an N50 of $776 \mathrm{bp}$. The average length of unigenes was $677.18 \mathrm{bp}$, and the longest sequence was $5815 \mathrm{bp}$. The sequence length distribution of the unigenes is shown in Additional file 1: Figure S1. Nearly half of the unigenes (49.03\%) ranged from 400 to $600 \mathrm{bp}$. The GC percentage was $42.73 \%$. All reads were deposited in NCBI and can be accessed in the Short Read Archive (SRA) under accession number SRX863944.

\section{Functional annotation of tree peony transcriptome}

We performed BLASTx (version 2.2.21) analysis against several protein databases: NCBI non-redundant (NR) protein, Swiss-Prot, Clusters of Orthologous Groups (COG), Gene Ontology (GO), and Kyoto Encyclopedia of Genes and Genomes (KEGG) using a cut off E-value of e-5 to annotate tree peony transcriptome. A total of 22,823 unigenes $(77.97 \%)$ were annotated in the NCBINR database based on sequence homology; 17,321 (59.17\%) were annotated in Swiss-Prot; 13,312 (45.47\%) were annotated in COG; 20,041 (68.46\%) were annotated in GO; and 9940 (43.55\%) were annotated in KEGG. In addition, 8070 (27.57\%) of the unigenes were annotated in the Pfam database. A total of 1939 unigenes were annotated in all databases, while 23,332 unigenes (79.7\%) were annotated in at least one database. It was found that the functional annotation of the $5815 \mathrm{bp}$ unigene was $26 \mathrm{~S}$ ribosomal RNA gene. The detailed results for annotation of the tree peony unigenes are summarized in Table 1.

Among the unigenes, 10,507 (33.35\%) unique sequences shared significant similarity with their matched sequences with an $E$ value ranging from $1 E$-60 to $1 E-10$. Only $30(0.13 \%)$ unique sequences shared weak similarity with the matched sequences ( $E$ value between $1 E-180$ and $1 E-190)$ (Fig. 1a). Further analysis showed that the annotated sequences were matched to sequences of 520 species. Among them, the highest matched species was Vitis vinifera and the matched unigenes were 9362 (27.84\%). The other top nine species were as follows: Theobroma cacao (6.42\%), Nelumbo nucifera (5.98\%), Jatropha curcas $(4.28 \%)$, Citrus $\times$ sinensis $(5.80 \%)$, Populus trichocarpa (3.38\%), Prunus mume (3.27\%),
Ricinus communis (3.13\%), Prunus persica (3.05\%), and Morus notabilis (2.65\%) (Fig. 1b).

To construct a shared protein domain with specific functions, 13,321 unigenes were grouped into 25 functional classifications based on the COG databases (Fig. 2). 'Signal transduction' was dominant (13.27\%), and the other top three functional groups were 'Posttranslational modification' (12.30\%), 'General function prediction only' (10.61\%), and 'RNA processing and modification' (6.95\%), respectively. 'Intracellular trafficking, secretion, and vesicular transport', 'Transcription', and 'Translation, ribosomal structure and biogenesis' shared $6.12,5.54$, and 5.19\% genes among the categories, respectively. The lowest matched term was 'Cell motility' and only had $0.017 \%$ corresponding genes.

The GO system alignment showed that these unigenes were classified into 63 main functional groups, belonging to biological process, cellular component, and molecular function, respectively (Fig. 3). In biological process, the vast majority was related to metabolic process, cellular process, and single-organism process. In cellular component, genes for cell, cell part, and organelle were the top three. Among the molecular function category, the majority of the GO terms were grouped into binding, catalytic activity, and transporter activity. The detailed information on the annotations was in Fig. 3.

Based on KEGG pathway mapping, we annotated and mapped 237 pathways for 9940 unigenes. A summary of the findings is presented in Fig. 4 and Additional file 2: Table S1. The largest number of sequences were those associated with metabolic pathways (1123, 11.30\%), followed by sequences that were involved in the biosynthesis of secondary metabolites (557, 5.045\%) and biosynthesis of antibiotics (276, 2.78\%). In particular, the plant circadian rhythm pathway was obtained using the KEGG database, and 26 genes were identified using the bud transcriptome (Additional file 3: Figure S2). It was suggested that the circadian rhythm was probably important for tree peony flowering.

\section{Differentially expressed genes (DEGs) identification and analysis through quantitative RNA-seq}

Investigating the gene expression level differences between different cultivars or the same cultivar in different developmental stages required identification of DEGs between any two samples. Expression levels of unigenes were determined by aligning the RNA-seq reads from

Table 1 The annotations of tree peony bud unigenes against the public databases

\begin{tabular}{llllllll}
\hline Database & NR & Swiss-Prot & COG & GO & KEGG & Pfam & All \\
\hline Number annotated & 22,823 & 17,321 & 13,312 & 20,041 & 9940 & 8070 & 29,275 \\
Percentage (\%) & $77.97 \%$ & $59.17 \%$ & $45.47 \%$ & $68.46 \%$ & $34.06 \%$ & $27.57 \%$ & 100 \\
\hline
\end{tabular}




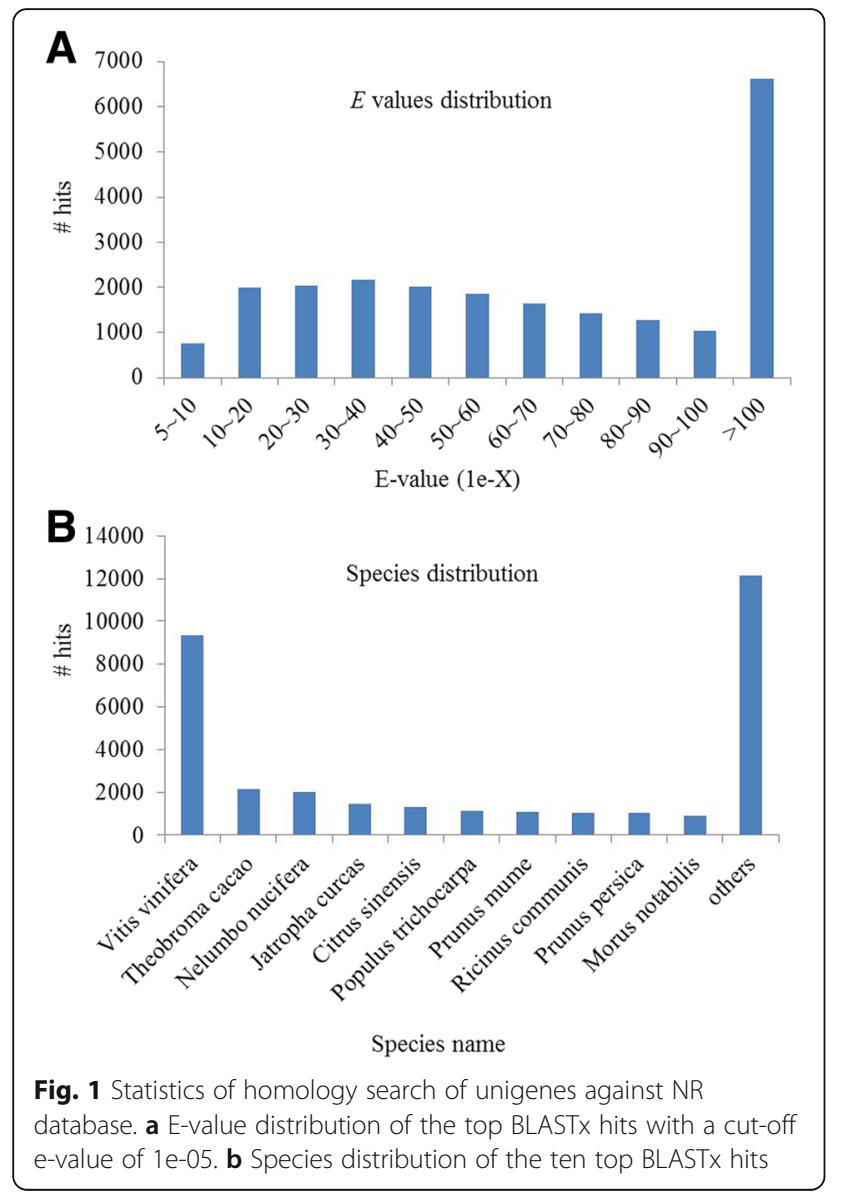

each library to the assembly. A $P$-value $<0.01, F D R \leq$ 0.001 , and $\log 2$ (fold change) $\geq 2$ or $\leq-2$ were used as thresholds to identify significant differences between two samples. Comparisons of gene expression in eight groups showed that 1297, 1348, 1484, 1395, 1636, 1058,
1383 and 1489 genes were differentially expressed in 'Huchuan Han' $(\mathrm{HCH})$ vs 'High Noon' $(\mathrm{HN}), \mathrm{HCH}$ vs 'Ziluo Lan' (ZLL), $\mathrm{HCH}$ vs Paeonia delavayi (PD), $\mathrm{HCH}$ vs 'Luoyang Hong' (LYH), HN vs PD, ZLL D (bud at stage D) vs ZLL, ZLL E (bud at stage E) vs ZLL, and ZLL E vs ZLL D, respectively. The detailed information of DEGs in eight groups is shown in Additional file 4: Figure S3, and the unigenes involved in different pathways are in Additional file 2: Table S1. The number of DEGs was largest in $\mathrm{HN}$ vs PD and smallest in ZLL D vs ZLL. The possible reason was that $\mathrm{HN}$ is a tree peony hybrid ( $P$. lutea $\mathrm{x} P$. suffruticosa). The most upregulated genes were in $\mathrm{HCH}$ vs $\mathrm{ZLL}$, while there were the fewest up-regulated genes in $\mathrm{HCH}$ vs PD. The most down-regulated genes were in $\mathrm{HCH}$ vs $\mathrm{PD}$, while the fewest down-regulated genes were in $\mathrm{HCH}$ vs ZLL (Additional file 4: Figure S3).

Further analysis of the up-regulated and downregulated genes in data from eight groups showed that flowering time genes, metabolism genes, and hormone synthesis and signal transduction genes had differential expression in different cultivars or developmental stages. Considering the flowering time character of four cultivars and one wild species, the flowering related genes were investigated further (Additional file 5: Table S2). In HCH vs HN, SVP, CONSTANS-LIKE 1 (COL1), VERNALIZATION INSENSITIVE 3 (VIN3), and AGAMOUS-LIKE 15 (AGL15) were down-regulated, while SPL5, GID2, ULTRAPETALA 1, and COL4 were upregulated. At the same time, FRIGIDA (FRI), blue-light photoreceptor PHR2, and gibberellin receptor GID1a genes appeared in both the down-regulated and upregulated groups. In $\mathrm{HCH}$ vs $\mathrm{PD}, C O L 1, A P E T A L A 2$ (AP2), PHR2, COL16, and SVP were down-regulated, while $F C A$ and GID1 were up-regulated. FRI and GID1a

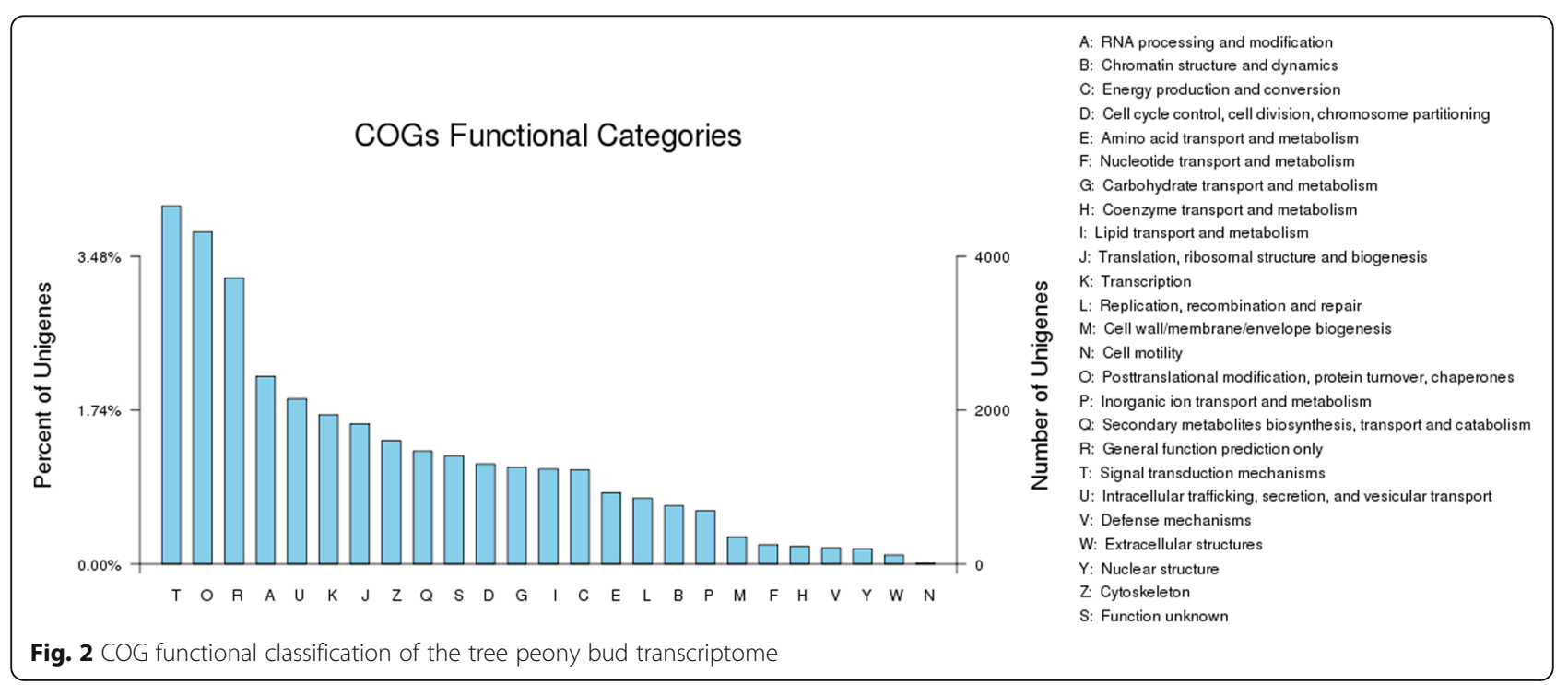




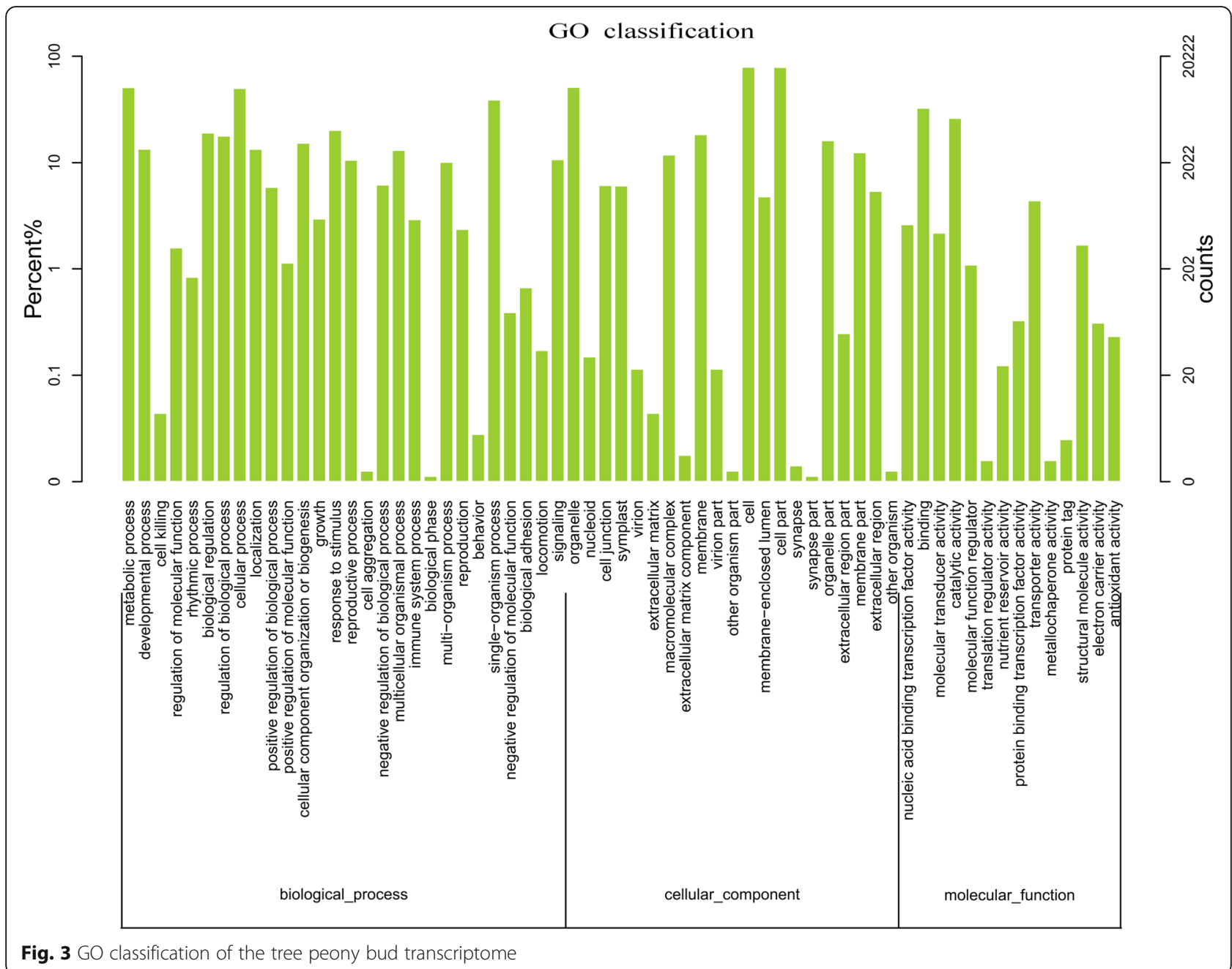

genes appeared in both down-regulated and upregulated groups. In $\mathrm{HCH}$ vs ZLL, the PHR2, SPL12, and GIGANTEA (GI) genes were down-regulated, while the Phytochrome E (PhyE), FRI, and AGL8O had upregulated expression. In $\mathrm{LYH}$ vs $\mathrm{HCH}$, the SPL12, SPL14, and Casein Kinase II (CKII) genes were downregulated, while AGL15, VIN3, EARLY Flowering 3, SPL16, and COL14 genes were up-regulated. FRI appeared in both down-regulated and up-regulated groups. In ZLL vs ZLL D, the AGL8 and AGL9 genes were down-regulated, while the FRI, CKII, and AGL80 genes were up-regulated. In ZLL vs ZLL E, GI, SPL14, LHY, and AGL9 were down-regulated, while the SPL14, FRI, and $C K I I$ genes were up-regulated. In ZLL D vs ZLL E, the AGL8 and SPL9 genes were down-regulated, while the FRI and COL4 genes were up-regulated. In $\mathrm{HN}$ vs PD, the GID1a, PhyE, AGL15, and SPL12 genes were down-regulated, while FRI and COL11 were upregulated. The COL4 gene was in both down-regulated and up-regulated groups. COL1, VIN3, and PsGI were the candidate re-blooming genes.

\section{Identification of putative genes involved in flowering time regulation}

Unlike in other model plants, the genetic network of flowering for tree peony is unclear. To identify the transcripts putatively involved in flowering time, flower meristem identity and flower organ identity of tree peony, previously reported flowering related genes in other model plant species, such as Arabidopsis thaliana, were used to search the transcripts database. In total, 64 flowering genes were identified in this work (Table 2). In addition, 13 important flowering genes with short sequences (length less than $200 \mathrm{bp}$ ) or those not identified by transcriptome sequencing were also isolated using bioinformatics methods, reverse transcript polymerase chain reaction (RT-PCR), and rapid-amplification of cDNA ends (RACE) (Table 2). These genes included flower organ identity genes (class A: $A P 1$ and $A P 2$, class B: $A P 3$ and PI, class C: $A G$, and class E: AGL9, SEP1, $S E P 3$, and SEP4); floral integrator pathway genes related to $F T, L F Y$, and $S O C 1$; floral meristem identity genes $C A L$ and $A P 1$; vernalization pathway genes related to 


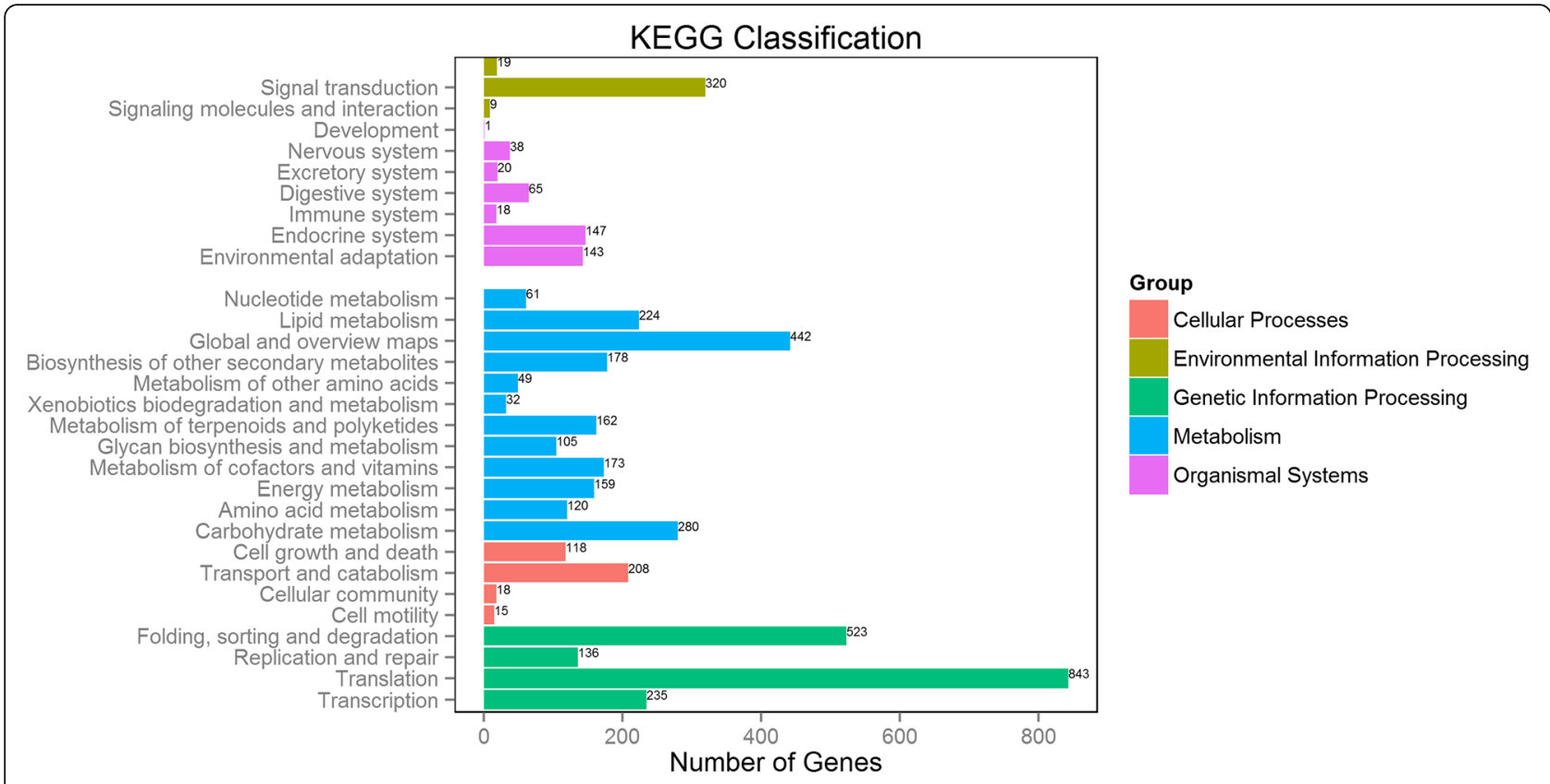

Fig. 4 KEGG classification of the tree peony bud transcriptome

HOS1-like, VIN3, VRN1, and VRN2; age pathway gene SPL9; GA pathway genes GAI, GID1, and SVP; autonomous pathway gene $F L D$; multiple genes responding to the photoperiod pathway, including $C O, C O L 4, C O L 6$, COL9, CRY1, CRY2, ELF3, ELF4, FKF1, LHY-like, PHYA, $P H Y B, P H Y C, P H Y E, W N K 1$, and ZTL; and floral repressor and promoter genes FRI, TFL, AG, and MAF-like.

\section{Relative expression analysis of DEGs related to flowering in the buds of four tree peony cultivars and one wild species}

To validate the results obtained from the differential gene expression and to determine the potential roles of the flowering genes referred above, we confirmed their expression in the buds of four cultivars and one wild species by qRT-PCR. Expression patterns of most of the DEGs were consistent with those obtained by RNA-seq, confirming the accuracy of the RNA-seq results reported in this study (Fig. 5, Additional file 5: Table S2). Those genes, including AP1, COL1, CRY1, GAI, LFY, LYH, and VIN3 had high expression in 'Ziluo Lan', which easily reblooms in autumn, together with leaf removal and $\mathrm{GA}_{3}$ application treatments. Genes including FT and SVP had high expression in 'Luoyang Hong', which does not easily flower in autumn. SOC1 and SPL9 had high expression in 'High Noon' which flowers in autumn under natural conditions. Combining the flowering characters of five tree peony cultivars, AP1, COL1, CRY1, FT, GI, LFY, LYH, SOC1, SPL9, SVP, and VIN3 were shown to be associated with tree peony autumn flowering or reblooming. It was deduced that tree peony flowering was regulated by GA, age, long day, and vernalization pathways.

In order to investigate whether the above genes played roles in flowering regulation, the key DEGs and previously reported key flowering time genes from the five pathways were chosen for gene expression analysis in different stages of differentiated primordium and developing buds (Figs. 6 and 7). Except for FT, GI, and TOC1, which were only highly expressed in the buds of stamen or/and pistil primordium stages, long day pathway genes including $C O L 2$, and $C R Y 2$, flowering integrator genes SOC1, LFY, and SVP, floral repressor gene FRI, vernalization pathway gene PsVIN3, gibberellin gene GID1, and aging pathway gene SPL9 were all highly expressed in buds of different stages of differentiated primordium. PsGI was highly expressed in the bud at stamen primordium stages (Fig. 6). These results indicated that all 12 genes may regulate bud differentiation, and that the time of regulation was different.

The expression patterns of the above genes were also detected in the buds from stage A (bud swelling) to stage $\mathrm{H}$ (color exposing) to detect the function of regulating flowering again. Generally, stages A to E are very important for tree peony flowering, especially for flowering of the forcing culture tree peony. Photoperiod related genes, such as COL2, CRY2, GI, and TOC1, and gibberellin gene PSGID1 had extremely high expression in big bell-like flower buds. Flowering integrator genes $F T$ and $L F Y$ were highly expressed in the buds at key stages (A to E, and H) (Fig. 7). Flowering repressor genes PSFRI and PSSVP had low 


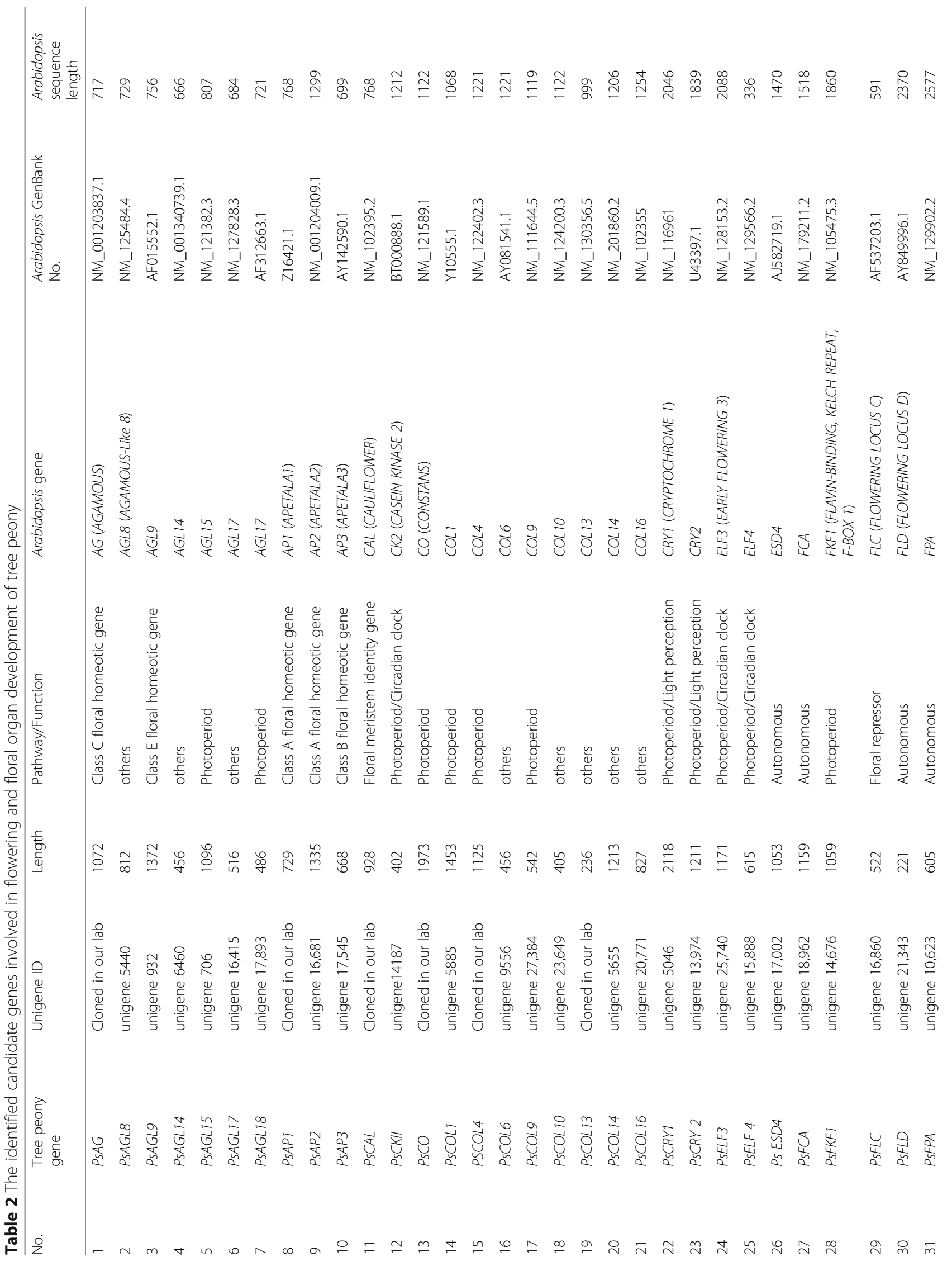




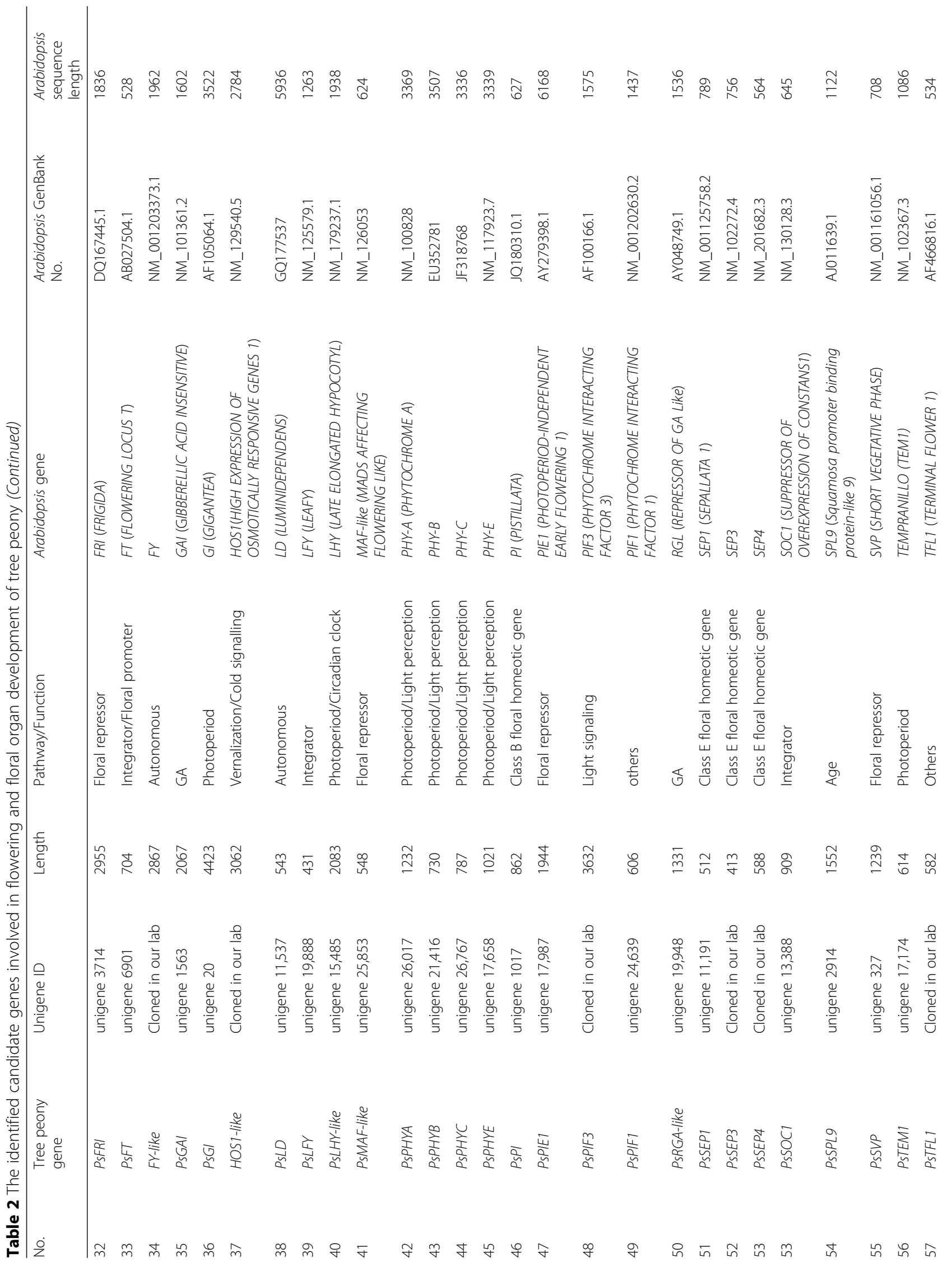




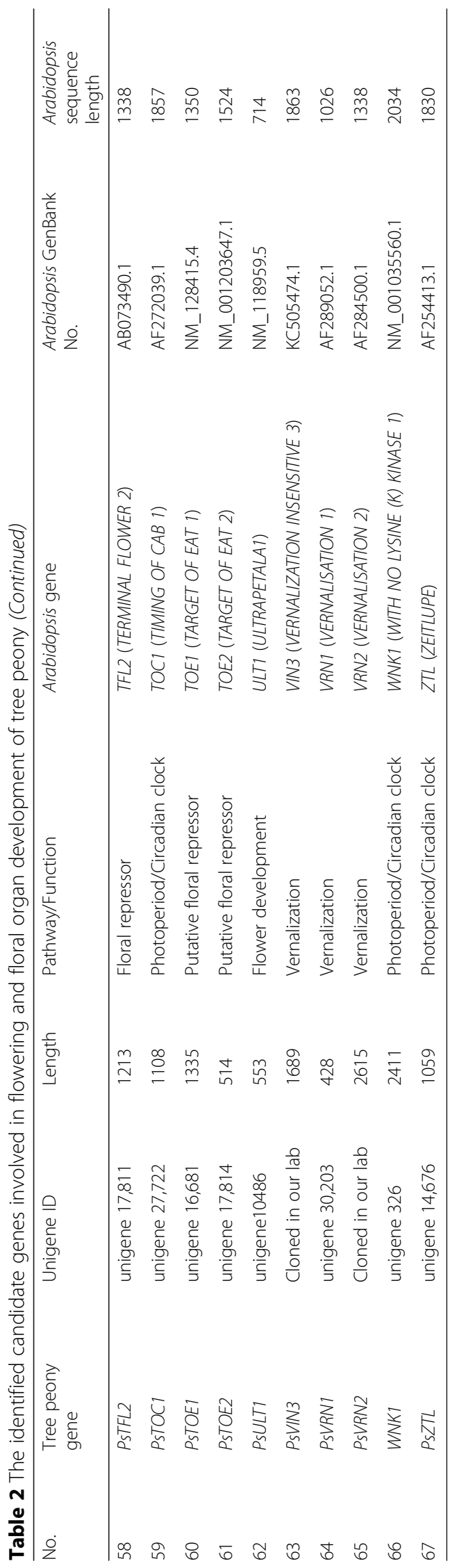




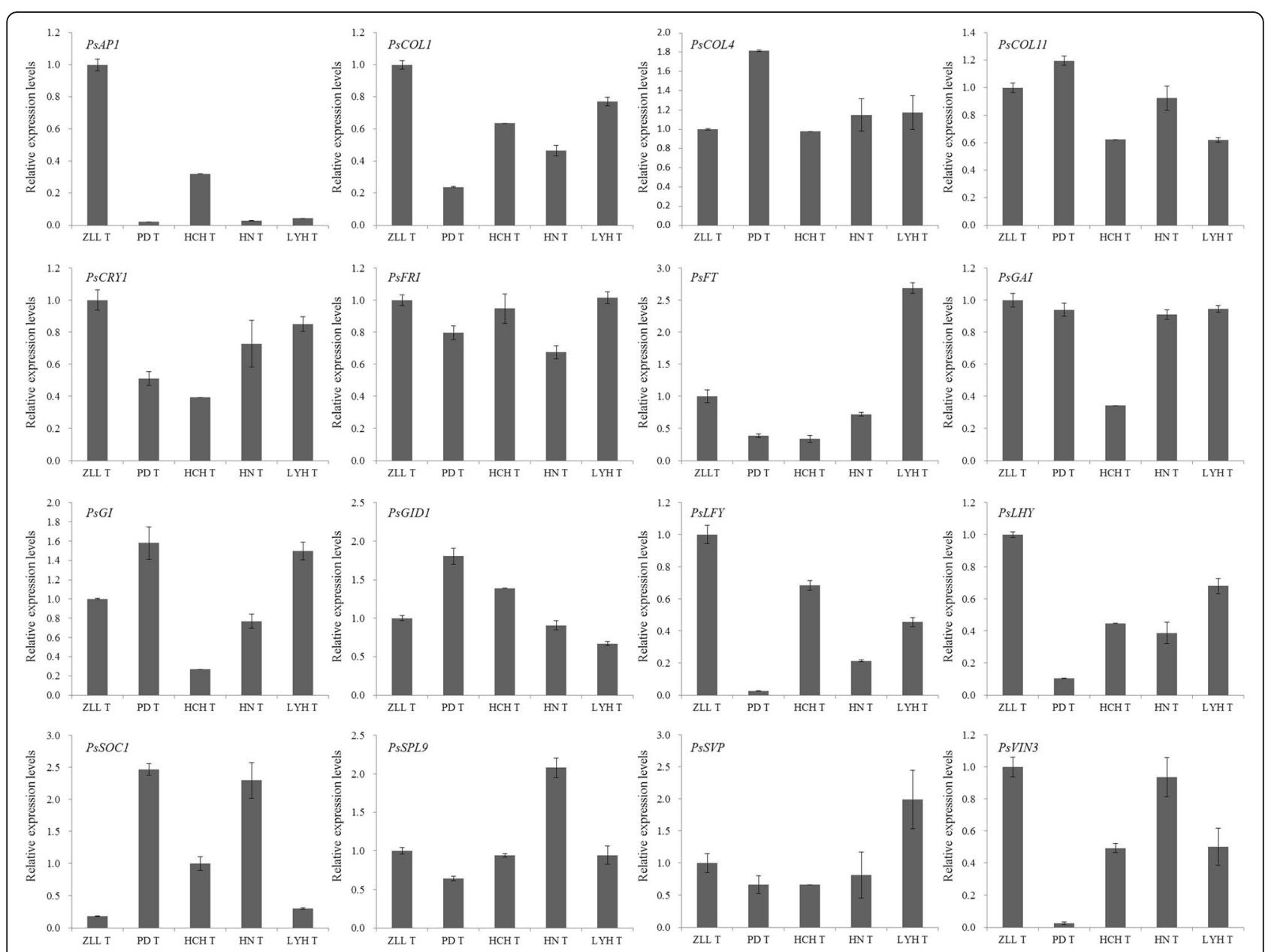

Fig. 5 The expression level validation of 12 DEGs in the buds of four cultivars and one wild species by qRT-PCR. ZLL T, PD T, HCH T, HN T, and LHY T represent the five samples used for transcriptome sequencing

expression in buds at stages $\mathrm{G}$ and $\mathrm{H}$ and had moderate expression in the buds from stages A to F (bigbell like stage) (Fig. 7); these genes are suspected to repress tree peony flowering. PSSPL9 had higher expression in the bud from stages A to $G$ and may also take part in flowering regulation and bud development in tree peony (Fig. 7). PsVIN3 also showed high expression in the eight different developmental buds (Fig. 7). The expression of SOC1 was highest in the sprouting bud and then decreased sharply and was slightly up-regulated in the bud from stages $\mathrm{F}$ to $\mathrm{H}$ (Fig. 7). These results suggested that PSSOC1 regulated flowering before bud swelling. Above all, long day, GA, age, and vernalization pathways were shown to be important for the flowering induction pathway in tree peony. The COL2, CRY2, GI, TOC1, PsGID1, FT, LFY, PsFRI, PsSVP, PsSPL9, PsVIN3, and PsSOC1 genes were the important genes in the flowering induction pathways.

\section{Expression analysis of key flowering genes in different treated buds}

In order to verify the four flowering induction pathways, treatments were designed for expression analysis of key flowering genes in the four pathways. Tree peony is long day plants, and the differentially expressed unigenes (Phy A, Phy B, FKF1, CRY, GI, LHY, FT, TOC1, etc.) were mainly involved in the circadian rhythm pathway (Additional file 3: Figure S2). This result indicated that the long day pathway is very important for regulating tree peony autumn flowering or re-blooming. Thus, phytochrome genes $C R Y 1$ and $C R Y 2$, clock entrainment genes $L H Y$ and $G I$, and flowering integrator gene SOC1 were chosen to do expression analysis in the first three developmental stages of buds in spring and autumn. Most of the genes had high expression in the spring buds (Fig. 8). In particular, the expression levels of PSCRY1 and PSCRY2 and floral integrator PSSOC1 were higher in buds in the spring than in autumn. These 

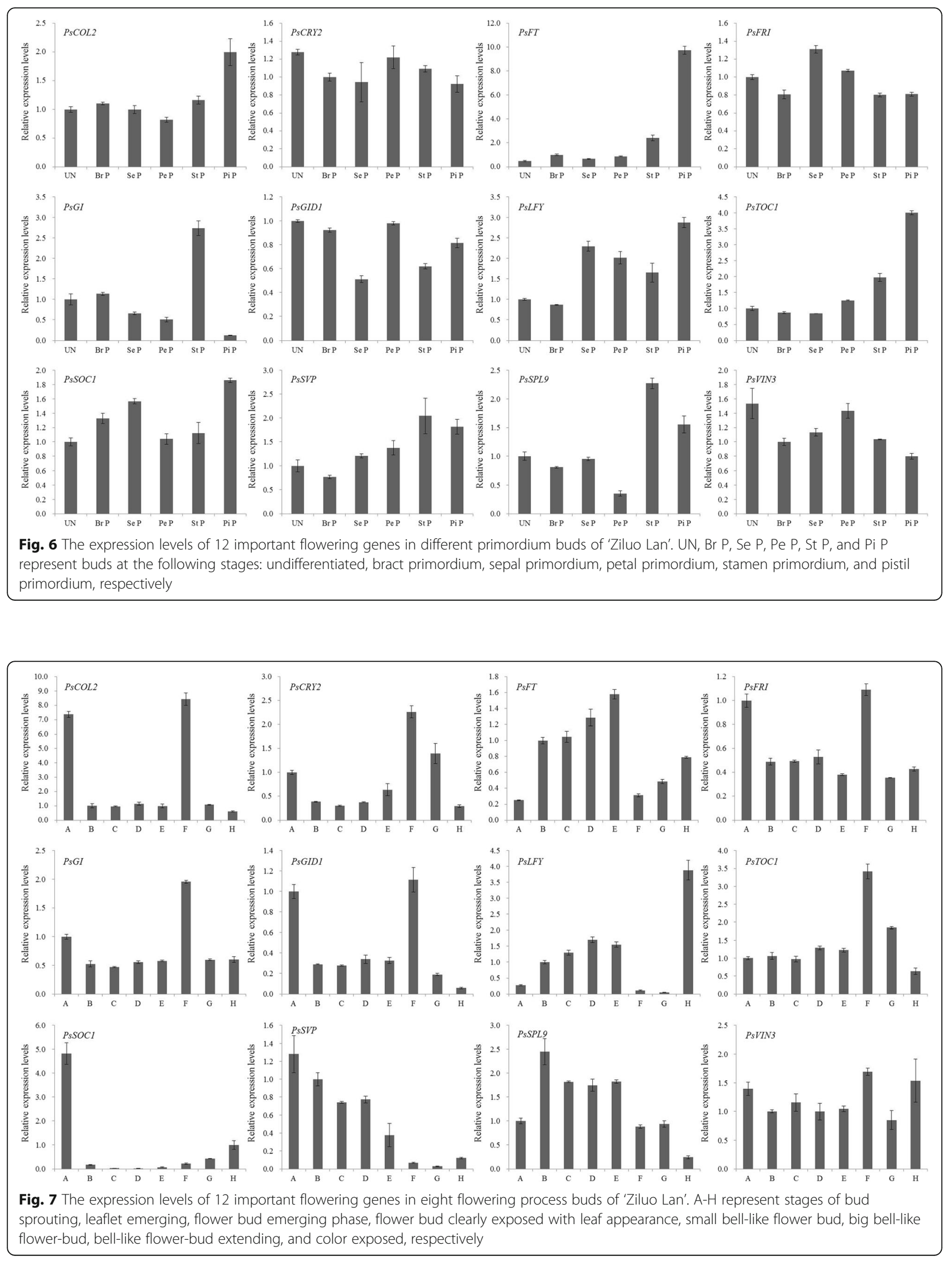

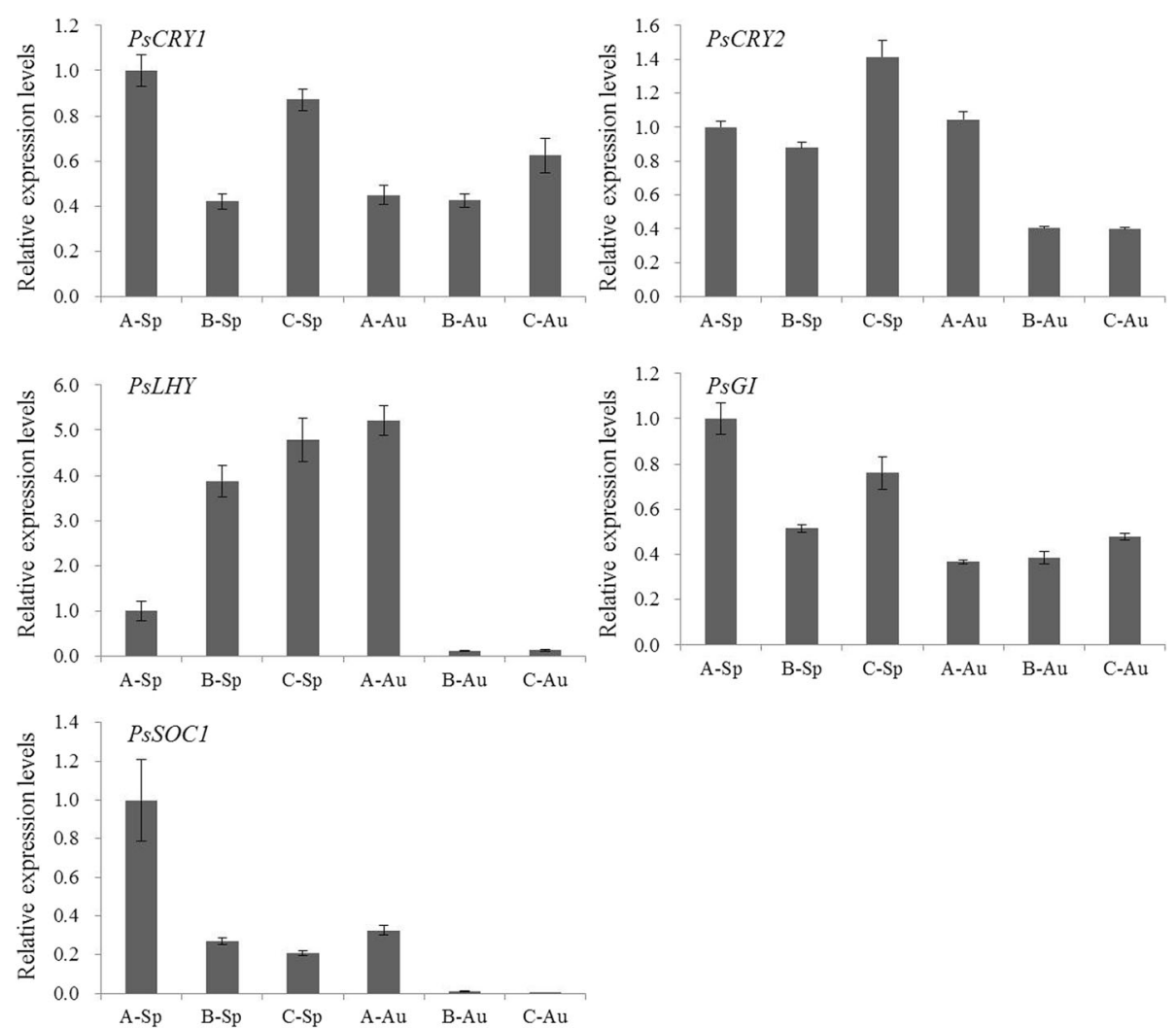

Fig. 8 The expression levels of flowering genes PSCRY1, PSCRY2, PSLHY, PSGI, and PSSOC1 in buds at the first three developmental stage of 'Ziluo Lan'. A, B, and C represent stages of bud sprouting, leaflet emerging, flower bud emerging phase, respectively, and Sp and Au represent spring and autumn, respectively

genes are important for plant flowering [9]. Considering the better flowering quality of spring compared to autumn, this result further indicated that the long day pathway was important for the flowering induction pathway.

The expression levels of PsAP1, PsFT, PsLFY, PsSOC1, and PsVRN3 could be up-regulated by vernalization treatment (Fig. 9). According to the gene function in the model plants, those genes were key genes regulating plant flowering, and higher expression of these genes may be induced by tree peony flowering. These upregulated genes also play important roles in regulating 'Ziluo Lan' re-blooming in autumn.

$\mathrm{GA}_{3}$ treatment results showed that $\mathrm{GA}_{3}$ promoted PSGAI and PSLFY expression in the treated buds after 1 week, and repressed SVP gene expression (Fig. 10). The expression levels of PsGID1 and PSSOC1 were promoted in buds $4 \mathrm{~h}$ after $\mathrm{GA}_{3}$ treatment and repressed after $1 \mathrm{e}$ week treatment (Fig. 10). PsGAI and PsGID1 are two important GA signaling genes. PsGID1 is upstream of PsGAI, and more PsGID1 expression will repress PsGAI expression [13]. The expression results of the two GA signaling genes were similar with the previous study
[13]. Endogenous $\mathrm{GA}_{3}$ could promote exogenous GA biosynthesis with $4 \mathrm{~h}$ treatment, and more biosynthetic GA induced PsGID1 expression and repressed PsGAI expression (Fig. 10). The expression levels of the flowering time genes were consistent with their functions. Expression results of those genes further validated that tree peony flowering could be induced by the GA pathway in the short day pathway rather than the long day pathway.

\section{Confirmation of differential expression of floral homeotic genes}

The eight identified floral homeotic genes including $A P 1, A P 2, A P 3, P I, A G, S E P 1, S E P 3$, and SEP4 were used to confirm their expression in different floral organs and different developmental stages of buds to determine their potential roles in floral organ development. The eight floral homeotic genes displayed distinctive spatial expression patterns in various floral organs (Fig. 11). $A P 1$ and $A P 2$ were predominantly expressed in the bract and sepal but had weak expression in petal and pistil and were hardly detected in stamen. In contrast, AP3 and $P I$ had strong expression in petal and stamen, but lower expression in pistil and sepal and were not 


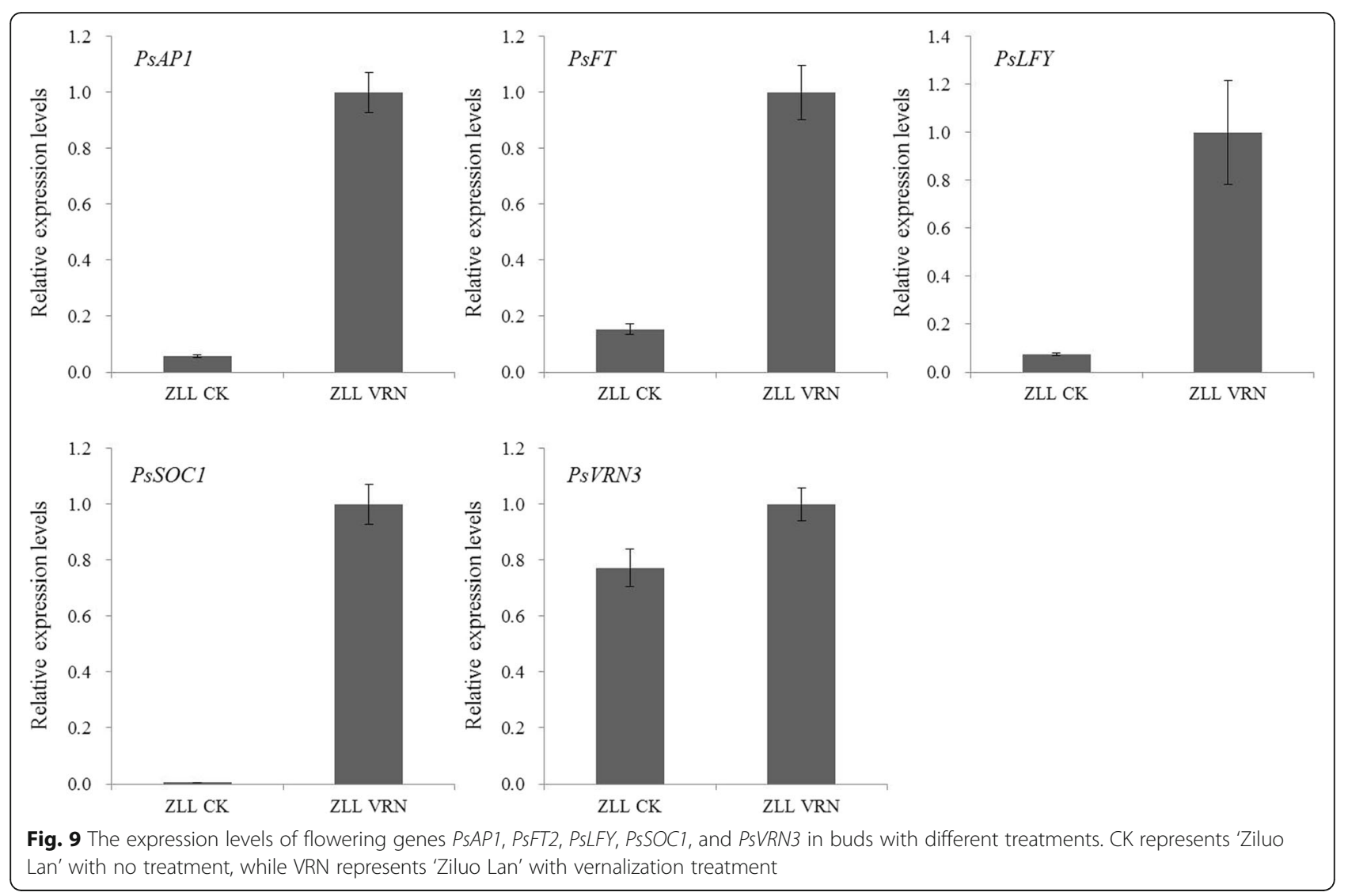

expressed in bract. $A G$ had high expression in stamen and pistil and lower expression in sepal and bract. Although the expression profiles of SEP1, SEP3, and SEP4 genes were different, they were expressed in the four whorls of flower organs. The SEP1 gene was preferentially expressed in sepal, stamen, and pistil; SEP3 was expressed in the buds of four different flower parts; and SEP4 had high expression in sepal and stamen. The above results suggested that $A P 1$ and $A P 2$ played roles in bract and sepal development; $A P 3$ and $P I$ regulated petal and stamen development; and $A G$ took part in stamen and pistil development. In addition, SEP1, SEP3, and SEP4 genes regulated development of the four whorls of floral organs.

In the different stages of differentiated flower buds, the expression patterns of $A P 1$ and $A P 2$ were opposite, with high expression of $A P 1$ in buds at the pistil primordium stage and high expression of AP2 in undifferentiated buds and buds at sepal and petal primordium stages (Fig. 11). Both AP3 and PI had high expression in the bud at the pistil primordium stage (Fig. 11). The $A G$ gene was expressed in all undifferentiated and differentiated buds and had especially high expression in the buds at the stamen and pistil primordium stages (Fig. 11). The SEP1, SEP3, and SEP4 genes were expressed in all the buds at four different differentiated stages (Fig. 11). These temporal expression results further confirmed that bract and sepal development were due to expression of class A genes such as AP1 and AP2, stamen development was due to the expression of class $\mathrm{B}$ genes $A P 3$, and $P I$, and class $C$ gene $A G$, and pistil development was due to the expression of class $C$ gene $A G$. Petal development was very complex most likely due to extremely abundant flower types of tree peony. The class E genes $S E P 1, S E P 3$, and SEP4 regulated the four whorls of floral organs development by interacting with class $\mathrm{A}, \mathrm{B}$, or $\mathrm{C}$ genes.

\section{Discussion}

Tree peony has large and colorful flowers that are valued globally. However, the short and relatively uniform flowering period is an important hindrance for tree peony production. Forcing cultures are often used to achieve year-round opening of ornamental peonies. However, little genomic information is available for this species, which limits the improvement of forcing culture technology. In this paper, we employed RNA-seq technology on buds of different tree peony cultivars to identify putative genes involved in flowering, floral organ development, and re- 

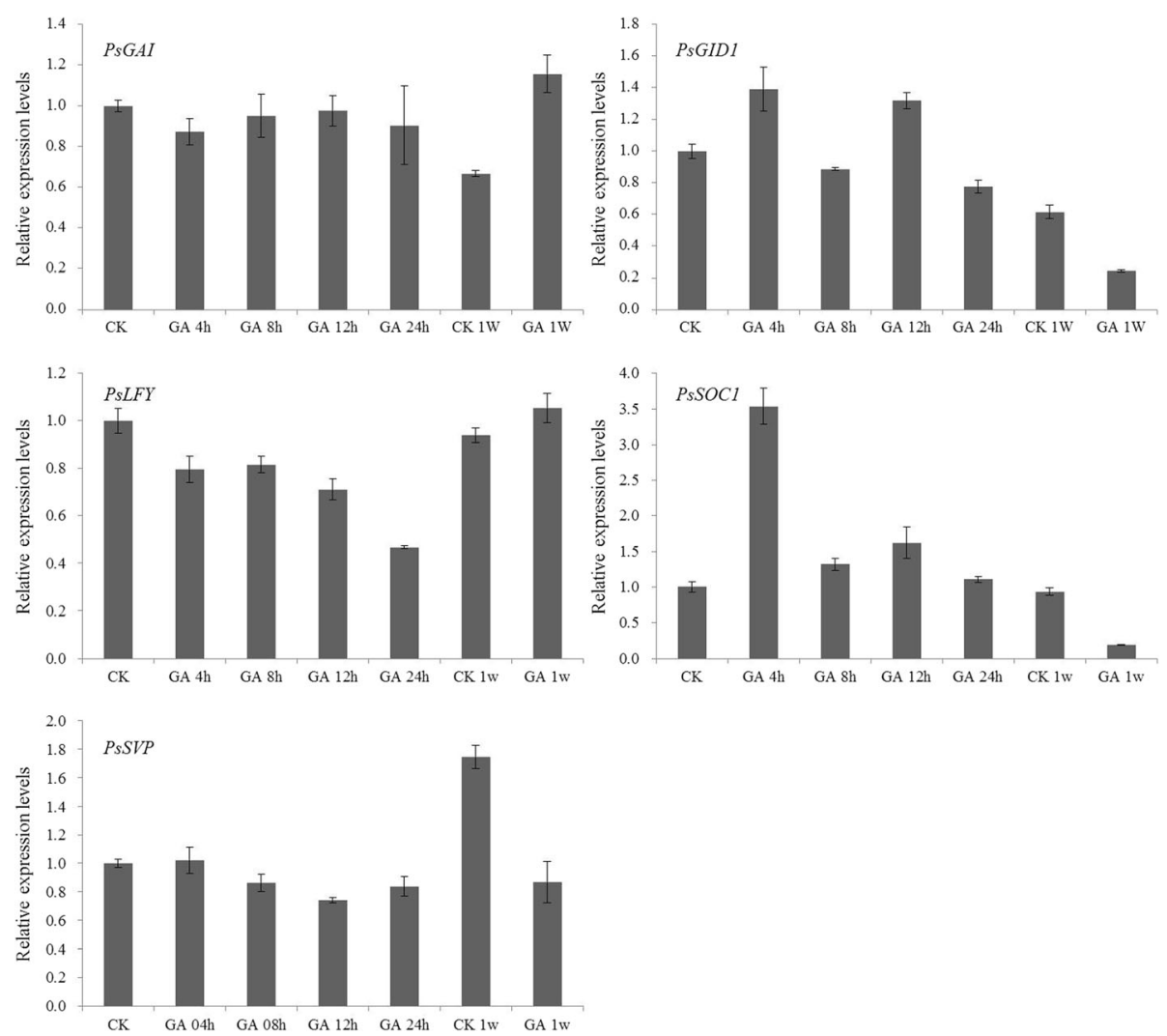

Fig. 10 The expression levels of GA synthesis genes PSGAl and PSGID1, and flowering time genes PSLFY, PSSOC1, and PSSVP in GA 3 treated buds of 'Ziluo Lan'. CK and CK $1 \mathrm{~W}$ represent buds after $0 \mathrm{~h}$ and 1 week without $\mathrm{GA}_{3}$ treatment, respectively, while GA $4 \mathrm{~h}, \mathrm{GA} 8 \mathrm{~h}, \mathrm{GA} 12 \mathrm{~h}, \mathrm{GA} 24 \mathrm{~h}$, and GA $1 \mathrm{~W}$ represents buds after $4 \mathrm{~h}, 8 \mathrm{~h}, 12 \mathrm{~h}, 24 \mathrm{~h}$ with $\mathrm{GA}_{3}$ treatment, respectively

blooming. Some important flowering genes were validated by qRT-PCR in buds at different developmental stages or treated buds to determine their function. The flowering induction pathway and the mechanism of flower organ development were proposed. These results will provide a theoretical basis for flowering regulation.

\section{Flowering habit and period of different tree peony cultivars}

Tree peonies have a long cultivation history in China and have been introduced to many countries starting in the Tang Dynasty [23]. Now, more than 2000 tree peony cultivars have been cultivated by long-term artificial selection and cross breeding $[1,2,23]$. Almost all of the cultivars flower once in the spring. Meanwhile, most spring flowering cultivars are mid-season peonies, and a smaller number of cultivars are early- and late-flowering [23]. The flowering habit of peonies has been artificially limited to extend the flowering period. However, some cultivars flower more than twice a year. American-group hybrid (P. lutea x P. suffruticosa) 'High Noon' not only flowers in spring, but also tends to re-bloom in autumn, and sometimes flowers again after the first flowering and before re-blooming in autumn [16]. A few intersectional hybrids ( $P$. suffruticosa $\mathrm{x} P$. latiflora) also have re-blooming character, with a late and long flowering period. The wild species $P$. delavayi, $P$. lutea, and $P$. potaninii are late-flowering, normally flowering in spring and can re-bloom at random in autumn [12]. Some Chinese cultivars 'Ziluo Lan', 'Bingzhao Lanyu', 'Chaoyang Hong' and 'Aoshuang' can re-bloom in the autumn $[11,25]$. Japanese cultivar 'Huchuan Han' and Chinese cultivar 'Luoyang Hong' were often used for forcing culture in winter $[1,2]$. The reasons for flowering habit and period in peony are unclear. Thus, understanding the mechanisms of regulating flower habit and period of peonies benefits forcing culture and breeding for flowering timing.

The large genome of tree peony (about $12.5 \mathrm{~Gb}$ ) coupled with a large amount of repetitive DNA has prevented genome sequencing projects in this species. De novo RNA-seq is often used to identify functional genes $[22,26]$. Thus, four tree peony cultivars with different flowering habit and period and one wild species were used to do RNA-seq to decipher the mechanism of 


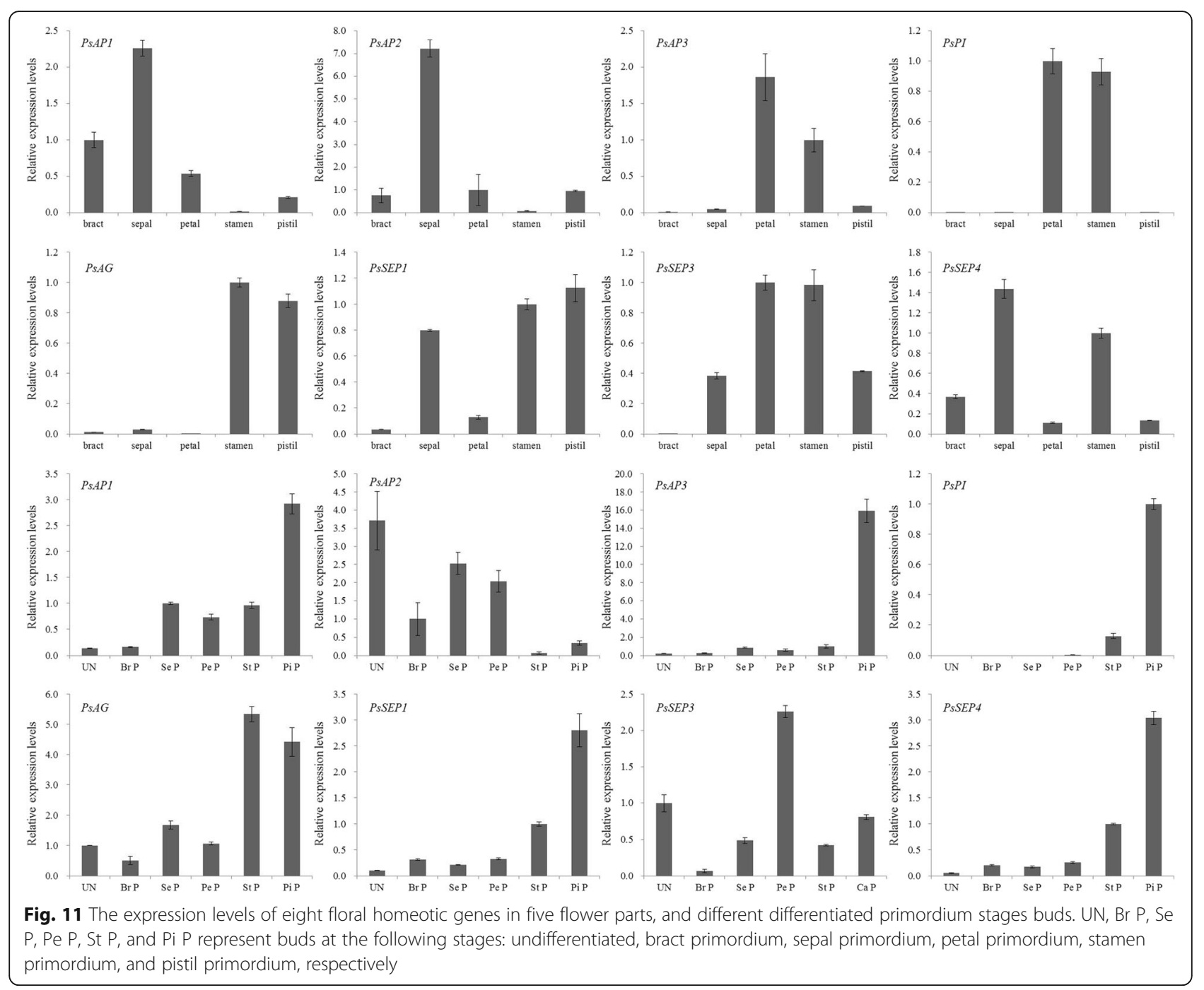

flowering regulation. Four hundred fifty four GS-FLX has many advantages for assembling and characterizing the gene space of a non-model species [27]. In this work, 31,505 contigs were assembled into 29,275 unigenes and 22,823 unigenes were annotated by NCBI-NR database. Compared with similar work reported in tree peony, the numbers and annotation information of unigenes was larger [15]. The average length of unigenes was longer than those reported in P. suffruticosa, Larix leptolepis, and Epinephelus coioides [15, 16, 27]. The highest matched species of the annotated sequences was $V$. vinifera, similar to that of $P$. ostii [15]. The sequence quality of 454 GSFLX was high in our work. Differentially expressed genes (DEGs) were found in different flower habit and period of tree peonies. The largest number of DEGs was found between American cultivar 'High Noon' and wild species $P$. delavayi. Different developmental buds of the same cultivar also had a large number of DEGs. These results show that the mechanism of 'High Noon' and $P$. delavayi may be different and that flowering is regulated by many important flowering genes.

\section{DEGs and putative schematic network of flowering induction pathways}

The flowering of tree peonies is a response to cues related to light, temperature, and other external influences $[1,2,12,13,25]$. Although the analysis of DEGs in reblooming and non-re-blooming cultivars, or early and late flowering period cultivars has identified some important flowering time genes and re-blooming genes using Illumina HiSeq ${ }^{\mathrm{Tm}} 2000$ and Illumina HiSeq ${ }^{\text {Tn }} 2500$ platforms $[15,16,23]$, the flowering induction pathway in tree peony remains unknown. Eight putative candidate genes of DEGs associated with floral induction, including PsCO, PsGI, PsFRI, PsVIN3, PsGA20ox, PsGID1, PSSOC1, and PSFT, were found in tree peonies [16]. These genes are involved in photoperiod, vernalization, and GA pathway. In this study, to comprehensively 
identify the candidate genes putatively implicated in flowering regulation in tree peony, a local BLASTx similarity search was performed against Arabidopsis and rice flowering genes from the NCBI database. Some flowering genes, such as FRI, CRY1, PHYA, TFL, and FVE were identified for the first time in tree peony. At the same time, some important flowering genes including $A G$, CAL, FY, LFY, HOS, and VIN3 were cloned by RACE or RT-PCR in our lab. Floral repressors including PSMAF1-4, PsTFL2, PsTOE1-2, and PSPIE1 and floral promoters $P S A G$ and PSMAF5 were characterized in our study. Finally, 67 flowering time genes involved in the flowering induction pathway, floral integrators, repressors, promoters, and organ development were obtained (Table 2), representing the most comprehensive report of flowering genes in tree peony.

In order to construct the schematic network of flowering regulation, the expression patterns of the flowering genes were determined. Expression levels of the flowering-related genes were compared in the buds of four tree peony cultivars, one wild species, and two developmental buds of 'Ziluo Lan' to determine the putative schematic network of flowering in tree peony
(Additional file 5: Table S2). According to different expression levels of those genes (Additional file 5: Table S2), and their functions in model plants [9], the completed schematic network of flowering induction pathways of tree peony was proposed. In tree peony, five pathways viz. long day, autonomous, vernalization, age, and gibberellin pathway regulated flowering (Fig. 12). However, the genes involved in the vernalization pathway did not show significant changes, except for PsFRI, based on DEGs analysis. The vernalization experiment showed that vernalization could significantly increase PSAP1, PsFT, PsLFY, PsSOC1, and PsVIN3 expression. By contrast, the number of DEGs was large in long day and autonomous pathways and it was deduced that long day and autonomous pathways were the two main flowering induction pathways. The expression levels of DEGs, such as GAI and GID1, in the GA pathway showed significant changes (Additional file 5: Table S2). Combining the results of effects of endogenous $\mathrm{GA}_{3}$ on flowering quality of 'Luoyang Hong' and the reblooming mechanism of 'High Noon', it was deduced that the GA pathway and vernalization pathway were also important pathways in tree peony $[13,16]$. PdSPL9

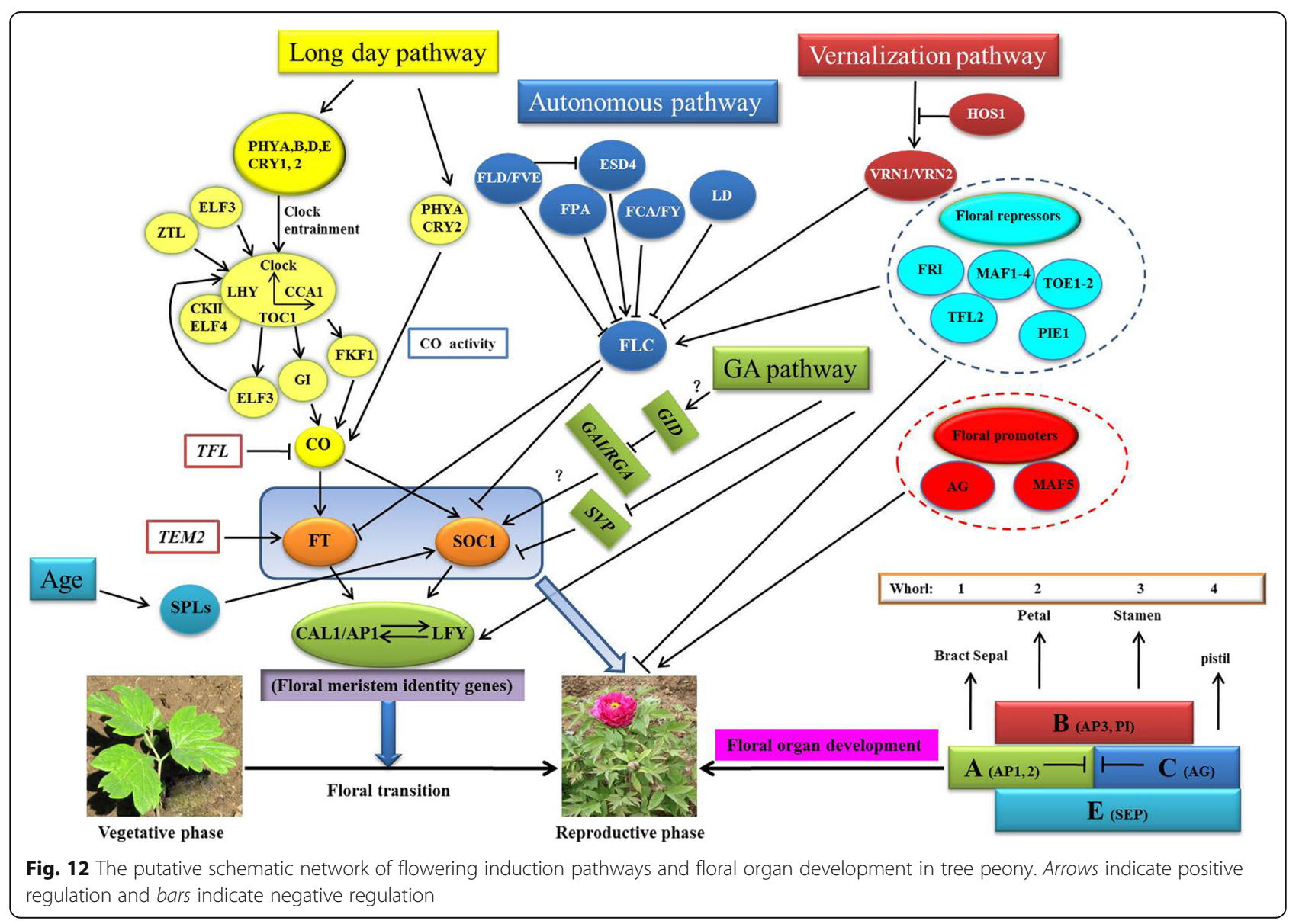


played important roles in the juvenile-to-adult phase transition, suggesting that the age pathway was also important in tree peony [12]. Above all, five main pathways, autonomous, long day, vernalization, age, and gibberellin regulated flowering in tree peony.

In order to verify the genetic network of the probable pathways, the important genes of the five pathways and floral integrators, repressors, and promoters were selected to do gene expression analysis in the different differentiated primordium and developmental buds (Figs. 6 and 7); the expression patterns of the above genes further confirmed the reliability of the flowering induction pathways of tree peony. Moreover, these results revealed that the known genetic flowering induction pathways and many critical flowering genes shared a high degree of conservation in tree peony, rice, radish, and Arabidopsis [4, 21, 28]. More flowering genes should be identified to improve the genetic network of flowering regulation in tree peony in the future.

\section{DEGs and the specification of flower organ development} Flower type is an important ornamental character, and there are ten flower types in tree peony. Stamen or pistil petalody results in increased whorls of petals and generates different flower types, which are one of the most important traits for cultivar classification [29]. However, the molecular mechanism of regulated floral organ development is still unclear. The family of MADS-box genes is a major group of regulators controlling floral transition, the specification of floral organ development, and regulating flowering time and other aspects of reproductive development [30, 31]. Our results also identified some members of MADS-box genes, such as AGLs, AP1, AP3, PI, and SEPALLATA1 (SEP1), which might participate in the specification of flower organ development (Table 2). The A-function homeotic gene $A P 2$ was also identified in this study and the C-function homeotic genes SEP3 and SEP4 were isolated by RACE and RT-PCR cloning in our lab (Table 2). All of the floral homeotic genes in tree peony were characterized in our study, providing valuable gene resources for flower type investigation.

In addition, the expression levels of eight homeotic genes were detected in the different flower parts and different stages of differentiated primordium and developmental buds to determine the gene function and floral organ development model. The bract covering the outside of the sepal was very large and unique in tree peony and the expression levels of the eight genes were investigated in the bract. The results revealed that eight genes had specific expression levels in five flower parts (Fig. 11). Results suggested that A-function genes including PSAP1 and PsAP2 determined sepal development and B-function genes including PSAP3 and PSPI determined petal development. PSAG was essential for stamen and pistil development, while PSSEP3 played important roles in development of the four whorls of floral organs. Development of the second whorl needed cooperated regulation by A-function genes, while third whorl development needed cooperated regulation of B-function genes. Based on these data, the genetic network of floral organ development in tree peony was postulated (Fig. 12). The floral organ development model of tree peony is an ABCE model, consistent with previous studies [32]. The bract development may be determined by A-function genes PSAP1, PsAP2, or Afunction genes + PSSEP4. The function of the PSSEP1 and PSSEP4 genes should be further investigated, as they are probably essential for abundant flower types and bract development. The alternative splicing of PhAGL6b was a key gene regulating specific labellum forming in Phalaenopsis [14]. Alternative splicing and variation of floral organ genes is also involved in abundant flower type forming in tree peony (data unpublished). Thus, the genetic regulation network of flower type is very complex. This postulated genetic network could provide a theoretical basis for tree peony flower type breeding.

\section{The important re-blooming genes of tree peony}

Re-blooming is very important for extending the flowering period and directly increasing economic benefits of ornamental tree peonies. Re-blooming genes were extensively investigated in the past 5 years $[1,2,16,23$, 25]. Four genes, PsCO, PsFT, PsGA20ox, and PsVIN3 probably play important roles in the regulation of the re-blooming process in tree peonies [16]. The expression patterns of GA biosynthesis and metabolism genes showed that PsGA20ox, PsGA2ox, and PsGA3ox were involved in the bioactive GAs synthesis, instead of directly operating in flowering [13]. The PsSVP and PsSOC1 genes are involved in flowering and vegetative growth of forcing culture tree peonies [1, 2]. PsCRY2 had higher expression in re-blooming 'Ziluo Lan' than that in non-re-blooming 'Luoyang Hong' and was increasingly expressed in the bud under long day conditions, compared short day conditions [25]. Previous studies and the expression patterns of the key flowering time genes in re-blooming and non-re-blooming tree peony, different stages of differentiated buds, flowering process, and vernalization experiment in this work, suggested that PsAP1, PsCOL1, PsCRY1, PsCRY2, PsFT, PsLFY, PsLHY, PsGI, PsSOC1, and PsVIN3 were the candidate re-blooming flowering genes. The most important re-blooming genes should be identified in the future. 


\section{Conclusions}

This work presents de novo transcriptome sequencing analysis of tree peony flower development using the 454 GS-FLX platform. A total of 29,275 unigenes were assembled with an average length of $677.18 \mathrm{bp}$, and 23,332 unigenes were annotated by at least one database among NCBI-NR, Swiss-prot, COG, GO, and KEGG. A total of 67 flowering-related genes were identified in tree peony, and the genetic regulation network of the flowering induction pathways and floral organ development were postulated. Moreover, the genes that regulated re-blooming in tree peony were proposed. Our work provides a theoretical basis for tree peony forcing culture and breeding for flowering period and flower type.

\section{Methods}

\section{Plant materials and sample collection for transcriptome} sequencing

In this work, transcriptome sequencing and gene expression analysis were performed on seven samples of tree peonies, including four cultivars and one wild species (Fig. 13). All of the cultivars and one wild species were introduced from Luoyang Tree Peony Gene Bank, China (there are no Genbank numbers, and only cultivar names in the Tree Peony Gene Bank), and grown in the Institute of Vegetables and Flowers Chinese Academy of Agricultural Sciences. Of these, 'Huchuan Han' with mid-flowering type, was from Japanese cultivars; 'High Noon' with late-flowering type, was from American cultivars; and 'Luoyang Hong', 'Ziluo Lan', and P. delevayi were Chinese cultivars or wild species and their flowering times were early-, mid- and late-flowering type, respectively. The cultivar names were referred to $\mathrm{Li}$ et al. [33] and the wild species was named by Abbe'Delavay for the first time $[3,33]$. The buds of the five samples (four cultivars and one wild species) were collected on 23-7-2012, and the other two samples, viz. clearly exposed buds and small bell-like flower bud of 'Ziluo Lan' were collected on 22-8-2012 and 12-9-2012, respectively. The seven samples for transcriptome sequencing were immediately frozen in liquid nitrogen and stored at $80^{\circ} \mathrm{C}$. 'Huchuan Han' can re-bloom in winter by forcing culture in Japan. 'High Noon' can re-bloom at autumn in natural conditions, while $P$. delevayi can re-bloom in autumn at random, and 'Ziluo Lan' can re-bloom in autumn with leaflet removal and $\mathrm{GA}_{3}$ application [2]. 'Luoyang Hong' was always used for tree peony forcing culture in winter; however, it could not easily re-bloom in autumn [1].

\section{Plant treatment and material collection}

In order to investigate the floral inductive pathways, floral organ development model, and mechanism of reblooming at autumn, 'Ziluo Lan' was selected to do different treatments. Detailed treatments were as follows:

(1) Sixty plants were selected to study the GA pathway. Leaves and extra buds were removed from half of the plants (only one to two buds were left for re-blooming) on 23-7-2012, and the remaining plants were as a control where only extra buds were removed. $\mathrm{GA}_{3}$ treatment was applied to buds at 6-8-2012, 8-8-2012, and 10-8-2012, respectively, to promote bud development and flowering. Buds were collected on 6-7-2012 and after GA treatment for $4 \mathrm{~h}$ and 1 week.

(2) Bud development was divided into eight stages, and the morphological developmental buds of 'Ziluo Lan' are in Additional file 6: Figure S4. A: Bud sprouting. In this stage flower buds tip emerged but was still covered by the scale. B: Leaflet emerging. The leaflet emerged but remained incurved. C: Flower bud emerging phase. Flower bud emerged and petiole extended, while the leaflet is still incurved. D: Flower bud clearly exposed with leaf appearance. Flower bud grows and its height is higher than that of leaflets. E: Small bell-like flower

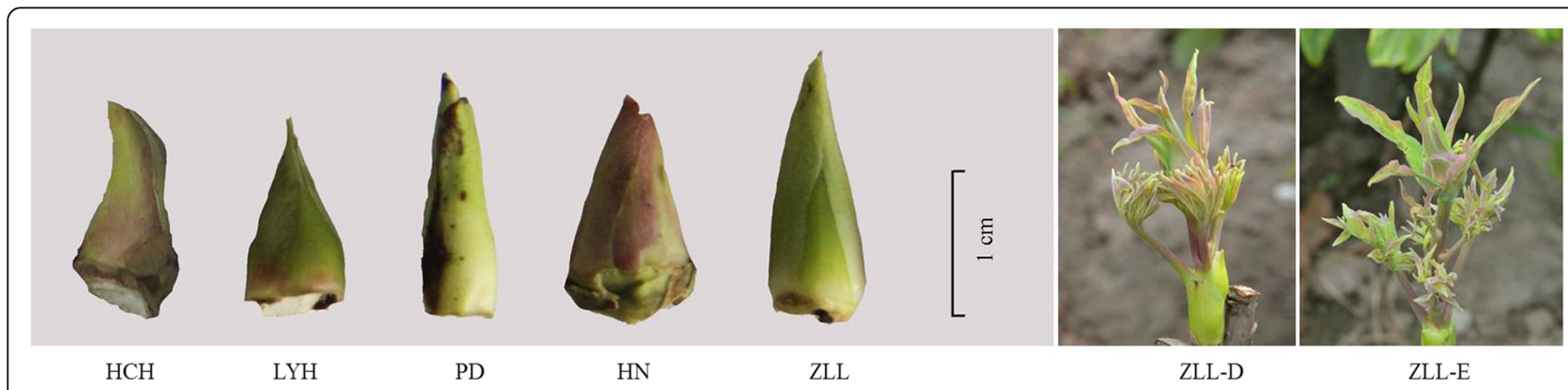

Fig. 13 The morphology of the seven samples used for transcriptome sequencing. HCH, LHY, PD, HN, ZLL, ZLL-D, and ZLL-E represent the peony names, 'Huchuan Han', 'Luoyang Hong', P. delavayi', 'High Noon', 'Ziluo Lan', clearly exposed buds of 'Ziluolan', and small bell-like flower bud of 'Ziluolan', respectively 
bud. Flower buds like a small bell. The leaves began unfolding and petiole opened outward. F: Big belllike flower-bud. Typical characteristics in this stage are that the flower bud enlarges, sepals become flat, and the leaf unfolds completely. G: Bell-like flowerbud extending. Enlarging flower bud turns large and tight. H: Color exposed. The colorful petal is exposed accompanied by loose and soft flower bud. Eight buds at different developmental stages were collected from 10 to 3-2013 to 28-4-2013. Buds at stages A, B, and C were also collected on 2-8-2012, 6-8-2012, and 9-8-2012, respectively, in the autumn by forcing culture treatments, to conduct re-blooming gene expression analysis. All the materials were cultivated in the field of the experimental base of the Institute of Vegetables and Flowers, Chinese Academy of Agricultural Sciences.

(3) Another 60 plants were selected to study vernalization. They were potted on 1-9-2012 and after 1 month of growth, half of the plants were stored in the refrigerator at $4{ }^{\circ} \mathrm{C}$ for one week; the remaining plants were stored in a $25^{\circ} \mathrm{C}$ greenhouse. Buds with vernalization treatment and control (CK) were collected. The morphology of buds could be seen in Additional file 7: Figure S5.

All three treatment buds were replicated from 2013 to 2014 and 2015-2016 for biological replicates.

(4) Five different differentiated primordium stages of buds were collected from 2013 to 6 to 2013-10 (Additional file 8: Figure S6).

(5) Five whorls of floral organs were collected on 2-5-2013.

\section{RNA extraction, construction of the CDNA library, and transcriptome sequencing}

For transcriptome sequencing, total RNA was extracted from seven samples using phenol-chloroform extraction. Concentration and purity of the total RNA was determined using a Nanodrop 1000 (Thermo Scientific, USA) . The mRNA was isolated and concentrated according to the instructions for the PolyATtract ${ }^{\circ}$ mRNA Isolation Systems (Promega, USA), and RNeasy RNA Cleaning Kit (QIAGEN, Germany), respectively. The mRNA integrity and quantity were assessed using an Agilent BioAnalyzer 2100 (Agilent Technologies, Santa Clara, CA). The firststrand cDNA and double-stranded cDNA (dscDNA) synthesis and dscDNA treatments were as in Zhang et al. [27]. Finally, the cDNA samples were processed with Roche 454 Genome Sequencer (GS) FLX Titanium General DNA Library Preparation Kit (Roche), following the manufacturer's instructions. Sequencing was carried out using a Roche 454 GS-FLX instrument. All the obtained data are available at the NCBI Short Read Archive (https://trace.ncbi.nlm.nih.gov/Traces/sra_sub/sub.cgi, accession number: SRX863944).

\section{De novo transcriptome assembly and annotation}

Raw data generated from 454 sequencing were preprocessed to remove the sequences of adapters, ambiguous nucleotides (' $\mathrm{N}$ ' in the end of reads) and low-quality sequences using LUCY software [34] and Seq-clean programs (http://sourceforge.net/projects/seqclean/). The screened high-quality sequences were subjected to de novo assembly using the Contig Assembly Program, CAP3, under default parameters [35]. Then, CD-HITEST was used to remove redundancy and retain the longest possible contigs. The short redundant contigs were removed and the remaining contigs composed the final unigenes for further analysis.

For annotation, the final unigenes were searched against the NCBI non-redundant (NR) protein database (2013.05) using BLASTx, with a cut-off E-value of $10^{-5}$ at first. Then, the final unigenes were used for BLASTx searches against the uniProt/Swiss-Prot protein database (2013.05). The unigene sequences were also aligned to the COG database ( $e$ value $<1.00 \mathrm{E}-05)$ to predict and classify functions. To understand the functional classification of the unigenes, gene ontology (GO) analysis was conducted on the annotated sequences using the Blast2GO Program [36]. In addition, to gain an overview of gene pathway networks, we carried out the Kyoto Encyclopedia of Genes and Genomes (KEGG) annotations based on the KEGG database.

\section{Differentially expressed genes (DEGs) analysis}

The Reads per Kilobase per Million mapped reads (RPKM) method was used to calculate the gene expression level [37]. Based on "the significance of digital gene expression profiles", differentially expressed genes (DEGs) between samples and their corresponding $P$-value were determined using methods described by Audic and Claverie [38]. The threshold of the $P$-value in multiple tests was determined by the value for the false discovery rate (FDR) [39]. FDR $\geq 0.001$ and the absolute value of $\log _{2} \mathrm{Ra}$ tio $\geq 1$ were used as the threshold to judge the significance of the gene expression differences.

\section{Quantitative real time PCR verification and expression analysis}

The extraction of total mRNA from different developmental stage buds, tissues, and organs, and buds with different treatments, mRNA purification, and cDNA synthesis were performed according to previously reported methods $[1,40]$. The DEGs and gene function prediction were performed by quantitative real time PCR (qRT-PCR). The 25 gene-specific primers were designed 
Table 3 List of primers for expression analysis of flowering genes

\begin{tabular}{|c|c|}
\hline Primer name & Sequences of the primers \\
\hline RTAG-2F & 5'-CAGGCAAATGTTGGGTGA-3' \\
\hline RTAG-2R & 5'-TGCTGGGCTCTITCGTTC-3' \\
\hline RTAP1-1F & 5'-AGAAGAAGGAAAGGGCAATC-3' \\
\hline RTAP1-1R & 5'-TTCCTCCTCACTTCTGTTGG-3' \\
\hline RTAP2-2F & 5'-CACGATGAATCCGATGACG-3' \\
\hline RTAP2-2R & 5'-GAAACCTCCACCGACTTGC-3' \\
\hline RTAP3.2-1F & 5'-TGGTGGAGAATGAGGGAG-3' \\
\hline RTAP3.2-1R & 5'-GGCGGAAAGCATACAAAT-3' \\
\hline RTCOL1-1F & 5'-AGGGCATTCAGTGAAGGAG-3' \\
\hline RTCOL1-1R & 5'-CCTACGCTCTTCAGTGGTG-3' \\
\hline RTCOL2-3F & 5'-GAGGCAAGAGTCCTAAGATACAG-3' \\
\hline RTCOL2-3R & 5'-AACCGCCCTTTGATTCGTG-3' \\
\hline RTCOL4-2F & 5'-TTGGTGAACGGAGGTGGT-3' \\
\hline RTCOL4-2R & 5'-TGAACTGCTGGATGATTTGT-3' \\
\hline RTCOL11-1F & 5'-GAAAAGAGGTGGAGACGAAG-3' \\
\hline RTCOL11-1R & 5'-AGACCACGGGACCACTTGA-3' \\
\hline RTCRY1-1F: & 5'-ACAACTTTCTCGGCATTCT-3' \\
\hline RTCRY1-1R & 5'-CAGCCTTTCTACGGTTCTT-3' \\
\hline RTCRY2-1F & 5'-CGTGCGAATAAAGCAGATA-3' \\
\hline RTCRY2-1R & 5'-GAAACAAAGGTATCGGGAG-3' \\
\hline RTFRI-2F & 5'-TCTTGCCACATTCGGTATT-3' \\
\hline RTFRI-2R & 5'-TCAGACAGGTCAAGGGAGC-3' \\
\hline RTFT-2F & 5'-CCAAGCGACCCAAACCTA-3' \\
\hline RTFT-2R & 5'-CGCCAACCTGGAGTGTAA-3' \\
\hline RTGAI-1F & 5'-GAGTATGCTGTCCGAGTTCA-3' \\
\hline RTGAI-1R & 5'-CAGGAGCAAGGAACGAAT-3' \\
\hline RTGI-1F & 5'-TAACCGCCCAATCTACAAG-3' \\
\hline RTGI-1R & 5'-ATTTCCCACAACACCGCTG-3' \\
\hline RTGID1-1F & 5'-TGAAGAACCTCCACCAAG-3' \\
\hline RTGID1-1R & 5'-CCACAAGACGACGACAAA-3' \\
\hline RTLFY-1F & 5'-ATGAGAAGGAAGGAGGGGATG-3' \\
\hline RTLFY-1R & 5'-CTTTGGCAATGGTCTGAACT-3' \\
\hline RTLHY-2F & 5'-GCAGTAACAGCGAGTGAGGT-3' \\
\hline RTLHY-2R & 5'-TTGCGGTAATACTTGTCGTGAG-3' \\
\hline RTSEP1-1F & 5'-TGAGCGTCAACTGGAAACAT-3 \\
\hline RTSEP1-1R & 5'-AGCAAGCTGATCGAGCATAT-3' \\
\hline RTSEP3-1F & 5'-TTGCGATGCGGAGGTTG-3' \\
\hline RTSEP3-1R & 5'-CCAAGGTCCTCACCAAGAAG-3' \\
\hline RTSEP4-1F & 5'-CTCTAACCGTGGGAAACTC-3' \\
\hline RTSEP4-1R & 5'-ACCTCTACCCTTGCCTTG-3' \\
\hline PsqSOC1-1F & 5'-CCAATGTCCGAGCAAGAAAG-3' \\
\hline PsqSOC1-1R & 5'-CCGTGCTTCTCGCATAACAT-3' \\
\hline RTSPL9-1F: & 5'-GGTTITGCCAGCAGTGTAGC-3' \\
\hline RTSPL9-1R & 5'-AGTCCATCAGAAAGCCTCCA-3' \\
\hline
\end{tabular}

Table 3 List of primers for expression analysis of flowering genes (Continued)

\begin{tabular}{ll}
\hline Primer name & Sequences of the primers \\
\hline RTSVP1-1F & 5'-CGATGTTGAGCAAGGAGGTT-3' \\
RTSVP1-1R & 5'-GCTCTAAATCAGCAGCGACA-3' \\
RT-TOC1-1F & 5'-AACTTGCGGCGTATTCCT-3' \\
RT-TOC1-1R & 5'-ATGCGTCTCCTCTCCAC-3' \\
RTVIN3-2F & 5'-GCAATCCAACGGAAGAAAGT-3' \\
RTVIN3-2R & 5'-AAGCAGCACAGCAGTAACCTC-3' \\
\hline
\end{tabular}

by primer 6.0 and the detailed information is shown in Table 3. The qRT-PCR program was outlined in Wang et al. [1, 2]. Relative expression levels of the candidate genes were calculated by normalizing to the reference gene ACTIN [1]. The qRT-PCR reaction was performed in three biological replicates, and three technical repetitions were performed for each replicate.

\section{Additional files}

Additional file 1: Figure S1. Sequence length distribution of the unigenes assembled from bud transcriptome sequencing. The horizontal and vertical axes show the size and the number of the unigenes, respectively. (JPG $29 \mathrm{~kb}$ )

Additional file 2: Table S1. The differentially expressed genes (DEGs) involved in the different pathways. (XLSX $77 \mathrm{~kb}$ )

Additional file 3: Figure S2. The unigenes involved in the plant circadian rhythm in bud of tree peony. The genes in red were found by our transcriptome sequencing. (PNG $19 \mathrm{~kb}$ )

Additional file 4: Figure S3. The differentially expressed genes based on comparisons of any two samples in bud transcriptome sequencing in tree peony. (JPG $186 \mathrm{~kb}$ )

Additional file 5: Table S2. The expression level analysis of the flowering-related genes in the seven samples by the Reads per Kilobase per Million mapped reads (RPKM) method. (XLSX 35 kb)

Additional file 6: Figure S4. The morphological characters of buds at eight different developmental stages of 'Ziluo Lan'. A: Bud sprouting. In this stage flower buds tip emerged but was still covered by the scale. B: Leaflet emerging. The leaflet emerged and remained incurved. C: Flower bud emerging phase. Flower bud emerged and petiole extended, while the leaflet is still incurved. D: Flower bud clearly exposed with leaf appearance. Flower bud grows and its height is higher than that of leaflets. E: Small belllike flower bud. Flower bud like a small bell. The leaves began unfolding and petiole opened outward. F: Big bell-like flower-bud. Typical characteristics in this stage are that flower bud enlarges, sepals become flat, and leaf unfolds completely. G: Bell-like flower-bud extending. Enlarging flower bud turned large and tight. $\mathrm{H}$ : Color exposed. The colorful petal is exposed accompanied by a loose and soft flower bud. (JPG $70 \mathrm{~kb}$ )

Additional file 7: Figure S5. The morphological characters of bud with or without vernalization. CK represents 'Ziluo Lan' with no treatment, while VRN represents 'Ziluo Lan' with vernalization treatment. (JPG 12 kb)

Additional file 8: Figure S6. The morphological characters of buds at six different differentiated primordium stages. UN, Br P, Se P, Pe P, St P, and $\mathrm{Pi} P$ represent buds at the following stages: undifferentiated, bract primordium, sepal primordium, petal primordium, stamen primordium, and pistil primordium, respectively. (JPG $126 \mathrm{~kb}$ )

\section{Abbreviations}

AGL: AGAMOUS-LIKE: AP1: APETALA1; CKII: Casein Kinase II: CO: CONSTANS. COG: Clusters of Orthologous Groups; DEGs: Differentially expressed genes; 
FLC: FLOWERING LOCUS C; FRI: FRIGIDA; FT: FLOWERING LOCUS T; GA: Gibberellins; GO: Gene ontology; HCH: Huchuan Han; HN: High Noon; KEGG: Kyoto Encyclopedia of Genes and Genomes; LFY: LEAFY; LYH: Luoyang Hong; NR: NCBI non-redundant protein; PD: Paeonia delavayi; PHYE: Phytochrome E; SEP1: SEPALLATA1; SOC1: SUPPERSSOR OF CONSTANS OF OVEREXPRESSION1; SPL: Squamosa promoter binding protein like; SPY: SPINDLY; SVP: SHORT VEGETATIVE PHASE; VIN3: VERNALIZATION INSENSITIVE 3; ZLL D: Bud at stage D of ZLL; ZLL E: Bud at stage D of ZLL; ZLL: Ziluo Lan

\section{Acknowledgements}

We would like to thank Dr. John Hugh Snyder for helping with improving the language of our manuscript.

\section{Authors' contributions}

SW designed and conducted experiments, analyzed data and drafted the manuscript, JG had done the vernalization and $\mathrm{GA}_{3}$ treatment of 'Ziluo Lan', $J X$ analyzed DEGs, YX collected the materials of experiments, DL analyzed the expression levels of the flowering related genes, YG improved Fig. 13 and Additional file 7: Figure S5, XZ supervised the experiment. All authors read and approved the final manuscript.

\section{Funding}

The work is supported by the grants from National Natural Science Foundation of China (31501800) and China Association for Science and Technology Foundation for Young Scholars (2015QRNC001), awarded to SW; and National Natural Science Foundation of China (31572156), China Agriculture Research System (CARS-21), and the Agricultural Science and Technology Innovation Program (ASTIP) of the Chinese Academy of Agricultural Sciences (CAAS-ASTIPIVFCAAS), awarded to XZ. The funding bodies had no role in the design of the study, the collection, analysis, and interpretation of data, or in writing the manuscript.

\section{Availability of data and materials}

All data generated or analyzed during this study are included in this published article and its supplementary information files. The nucleotide sequences of raw data from this study were submitted to the NCBI sequence read Archive (SRA) under the accession number SRX863944.

\section{Ethics approval and consent to participate}

Not applicable.

\section{Consent for publication}

Not applicable.

\section{Competing interests}

The authors declare that they have no competing interests.

\section{Received: 5 April 2019 Accepted: 29 May 2019}

Published online: 11 July 2019

\section{References}

1. Wang SL, Xue JQ, Ahmadi N, Holloway P, Zhu FY, Ren XX, Zhang XX. Molecular characterization and expression patterns of PSSVP genes reveal distinct roles in flower bud abortion and flowering in tree peony (Paeonia Suffruticosa). Can J Plant Sci. 2014a;94:1181-93.

2. Wang SL, Beruto M, Xue JQ, Zhu FY, Liu CJ, Yan YM, Zhang XX. Molecular cloning and potential function prediction of homologous SOC1 genes in tree peony. Plant Cell Rep. 2015;34:1459-71.

3. Zhou SL, Zou XH, Zhou ZQ, Liu J, Xu C, Yu J, Wang Q, Zhang DM, Wang XQ, Ge S, Sang T, Pan KY, Hong DY. 2014. Multiple species of wild tree peonies gave rise to the 'king of flowers', Paeonia suffruticosa Andrews. Proc R Soc 2014. 281: 20141687.

4. Simpson GG, Gendall AR, Dean C. When to switch to flowering. Ann Rev Cell Dev Biol. 1999;99:519-50.

5. Seo E, Lee H, Jeon J, Park H, Kim J, Noh YS, Lee I. Crosstalk between cold response and flowering in Arabidopsis is mediated through the flowering time gene SOC1 and its upstream negative regulator FLC. Plant Cell. 2009;21:3185-97.

6. Wellmer F, Riechmann JL. Gene networks controlling the initiation of flower development. Trends Genet. 2010;26(12):519-27.
7. Wang JW. Regulation of flowering time by the miR156-mediated age pathway. J Exp Bot. 2014b;65(17):4723-30.

8. Li B, Xiao G, Luo K, Wang Z, Mao B, Lin X, Guo X. Overexpression of PVGF14C from Phyllostachys violascens delays flowering time in transgenic Arabidopsis. Front Plant Sci. 2018;9:105.

9. Lee J, Lee I. Regulation and function of SOC1, a flowering pathway integrator. J Exp Bot. 2010;61:2247-54.

10. Hosoki T, Hamada M, Maeda T, Gotoh T. Forcing of tree peony for december shipping using spring- and winter-blooming cultivars. J Jpn Soc Horticultural Sci. 1992;61:121-6.

11. Mornya PMP, Cheng FY. The levels of hormone and carbohydrate in autumn and non-autumn flowering tree peonies. Can J Plant Sci. 2011;91:991-8.

12. Zhu FY, Wang SL, Xue JQ, Li DD, Ren XX, Xue YQ, Zhang XX. Morphological and physiological changes, and the functional analysis of PdSPL9 in the juvenile-to-adult phase transition of Paeonia delavayi. Plant Cell Tissue Organ Cult. 2018;133:325-37.

13. Guan YR, Xue JQ, Xue YQ, Yang RW, Wang SL, Zhang XX. Effect of exogenous $\mathrm{GA}_{3}$ on flowering quality, endogenous hormones, and hormone-, and flowering-associated gene expression in forcing-cultured tree peony (Paeonia suffruticosa). J Integr Agric. 2018;17:60345-7.

14. Huang X, Xue TT, Dai SL, Gai SP, Zheng CC, Zheng GS. Genes associated with the release of dormant buds in tree peonies. Acta Physiol Plant. 2008;30:797-806.

15. Gai SP, Zhang YX, Mu P, Liu CY, Liu S, Dong L, Zheng SS. Transcriptome analysis of tree peony during chilling requirement fulfillment: assembling, annotation and markers discovering. Gene. 2012;497:256-62.

16. Zhou H, Cheng FY, Wang R, Zhong Y, He CY. Transcriptome comparison reveals key candidate genes responsible for the unusual reblooming trait in tree peonies. PLoS One. 2013;8(11):e79996.

17. Wang Z, Gerstein M, Snyder M. RNA-Seq: a revolutionary tool for transcriptomics. Nat Rev Genet. 2009;10:57-63.

18. Liu DF, Sui SZ, Ma J, Li ZN, Guo YL, Luo DP, Yang JF, Li MY. Transcriptomic analysis of flower development in wintersweet (Chimonathus praecox). PLoS One. 2015a;9:e86976.

19. Liu XM, Dong YY, Yao N, Zhang Y, Wang N, Cui XY, Li XW, Wang YF, Wang FW, Yang J, Guan LL, Du LN, Li HY, Li XK. De novo sequencing and analysis of the safflower transcriptome to discover putative genes associated with safflor yellow in Carthamus tinctorius L. Int J Mol Sci. 2015b;16:25657-77.

20. Li WQ, Liu XH, Lu YM. Transcriptome comparison reveals key candidate genes in response to vernalization of oriental lily. BMC Genomics. 2016;17:664

21. Nie SS, Li C, Xu L, Wang Y, Huang DQ, Muleke EM, Sun XC, Xie Y, Liu LW. De novo transcriptome analysis in radish (Raphanus sativus L.) and identification of critical genes involved in bolting and flowering. BMC Genomics. 2016;17:389.

22. Huang JZ, Lin CP, Cheng TC, Huang YW, Tsai YJ, Cheng SY, Chen YW, Lee CP, Chung WC, Chang BCH, Chin SW, Lee CY, Chen FC. The genome and transcriptome of Phalaenopsis yield insights into floral organ development and flowering regulation. Peer J. 2016;4:e2017.

23. Hou XG, Guo Q, Wei WQ, Guo LL, Guo DL, Zhang L. Screening of genes related to early and late flowering in tree peony based on bulked segregant RNA sequencing and verification by quantitative real-time PCR. Molecules. 2018;23:689.

24. Collins LJ, Biggs PJ, Voelckel C, Joly S. An approach to transcriptome analysis of non-model organisms using short-read sequences. Genome Inform. 2008;21:3-14.

25. Ren XX, Wang SL, Xue JQ, Zhu FY, Liu CJ, Zhang XX. Molecular cloning and expression analysis of cryptochrome gene PSCRY2 in tree peony. Horticultural Plant J. 2016;2(6):357-62.

26. Xu HM, Kong XD, Chen F, Huang JX, Lou XY, Zhao JY. Transcriptome analysis of Brassica napus pod using RNA-Seq and identification of lipidrelated candidate genes. BMC Genomics. 2015;16:858.

27. Zhang Y, Zhang SG, Han SY, Li XM, Qi LW. Transcriptome profiling and in silico analysis of somatic embryos in Japanese larch (Larix leptolepis). Plant Cell Rep. 2012;31:1637-57.

28. Shrestha R, Gómez-Ariza J, Brambilla V, Fornara F. Molecular control of seasonal flowering in rice, Arabidopsis and temperate cereals. Ann Bot. 2014;114:1445-58.

29. Shu QY, Wang LS, Wu J, Du H, Liu ZA, Ren HX, Zhang JJ. Analysis of the formation of flower shapes in wild species and cultivars of tree peony using the MADS-box subfamily gene. Gene. 2011;493:113-23. 
30. Becker A, Theißen $G$. The major clades of MADS-box genes and their role in the development and evolution of flowering plants. Mol Phylogenet Evol. 2003;29:464-89.

31. Dornelas MC, Patreze CM, Angenent GC, Immink RG. MADS: the missing link between identity and growth? Trends Plant Sci. 2011;16(2):89-97.

32. Causier B, Schwarz-Sommer Z, Davies B. Floral organ identity: 20 years of ABCs. Semin Cell Dev Biol. 2010;21:73-9.

33. Li J. Tree peony of China. Encyclopedia of China Publishing House 2011. 1-324.

34. Chou HH, Holmes MH. DNA sequence quality trimming and vector removal. Bioinformatics. 2001;17:1093-104.

35. Huang X, Madan A. CAP3: a DNA sequence assembly program. Genome Res. 1999;9(9):868-77.

36. Conesa A, Götz S. Blast2GO: a comprehensive suite for functional analysis in plant genomics. Int J Plant Genomics. 2008:1-12.

37. Mortazavi A, Williams BA, McCue K, Schaeffer L, Wold B. Mapping and quantifying mammalian transcriptomes by RNA-Seq. Nat Methods. 2008;:5:621-8.

38. Audic S, Claverie JM. The significance of digital gene expression profiles. Genome Res. 1997;7:986-95.

39. Benjamini $Y$, Yekutieli $D$. The control of the false discovery rate in multiple testing under dependency. Ann Stat. 2001;29:1165-88.

40. Wang SL, Shen XX, Ge P, Li J, Subburaj S, Li XH, Zeller FJ, Hsam SLK, Yan YM. Molecular characterization and dynamic expression patterns of two types of Y-gliadin genes from Aegilops and Triticum species. Theor Appl Genet. 2012; 125:1371-84.

\section{Publisher's Note}

Springer Nature remains neutral with regard to jurisdictional claims in published maps and institutional affiliations.

Ready to submit your research? Choose BMC and benefit from:

- fast, convenient online submission

- thorough peer review by experienced researchers in your field

- rapid publication on acceptance

- support for research data, including large and complex data types

- gold Open Access which fosters wider collaboration and increased citations

- maximum visibility for your research: over $100 \mathrm{M}$ website views per year

At BMC, research is always in progress.

Learn more biomedcentral.com/submissions 